\title{
Heterogeneity induced GZMA-F2R communication inefficient impairs antitumor immunotherapy of PD-1 mAb through JAK2/ STAT1 signal suppression in hepatocellular carcinoma
}

Yuxue Gao ${ }^{1}$, Qingguo Xu ${ }^{2}$, Xinqiang $\mathrm{Li}^{2}$, Yuan Guo ${ }^{2}$, Bowen Zhang ${ }^{3}$, Yan $\mathrm{Jin}^{2}$, Cunle zhu ${ }^{2}$, Yuntai Shen ${ }^{2}$, Pengxiang Yang ${ }^{1}$, Ying Shi ${ }^{1}$, Rifeng $\mathrm{Jin}^{4}$, Daojie Liu ${ }^{5}$, Yabo Ouyang ${ }^{1}$, Xiaoni Liu ${ }^{1}$, Wenjing Wang ${ }^{1}$, Dexi Chen ${ }^{10}{ }^{1,2}$ and Tongwang Yang (iD) ${ }^{1,2}$

(c) The Author(s) 2022

Tumor heterogeneity has been associated with immunotherapy and targeted drug resistance in hepatocellular carcinoma (HCC). However, communications between tumor and cytotoxic cells are poorly understood to date. In the present study, thirty-one clusters of cells were discovered in the tumor tissues and adjacent tissues through single-cell sequencing. Moreover, the quantity and function exhaustion of cytotoxic cells was observed to be induced in tumors by the TCR and apoptosis signal pathways. Furthermore, granzyme failure of cytotoxic cells was observed in HCC patients. Importantly, the GZMA secreted by cytotoxic cells was demonstrated to interact with the F2R expressed by the tumor cells both in vivo and in vitro. This interaction induced tumor suppression and T cell-mediated killing of tumor cells via the activation of the JAK2/STAT1 signaling pathway. Mechanistically, the activation of JAK2/STAT1 signaling promoted apoptosis under the mediating effect of the LDPRSFLL motif at the N-terminus of F2R, which interacted with GZMA. In addition, GZMA and F2R were positively correlated with PD-1 and PD-L1 in tumor tissues, while the expressions of F2R and GZMA promoted PD-1 mAb-induced tumor suppression in both mouse model and HCC patients. Finally, in HCC patients, a low expression of GZMA and F2R in the tumor tissues was correlated with aggressive clinicopathological characteristics and poor prognosis. Collectively, GZMA-F2R communication inefficient induces deficient PD-1 mAb therapy and provide a completely novel immunotherapy strategy for tumor suppression in HCC patients.

Cell Death and Disease (2022)13:213; https://doi.org/10.1038/s41419-022-04654-7

\section{INTRODUCTION}

T cells are strictly controlled via the presentation of ligands and their co-stimulatory and co-inhibitory receptors for the maintenance of self-tolerance during tumor suppression [1]. Immune checkpoints are widely recognized for their role in tumor suppression based on the outstanding outcomes reported for PD-1 and PD-L1 mAb [2-5]. Unfortunately, only a limited number of patients have demonstrated long-term responses to the therapies based on immune checkpoints $[6,7]$.

Studies have suggested a heterogeneous character of tumors $[8,9]$, with several differences existing among different types of primary tumors and also between primary tumors and metastatic tumors [10]. In addition, polyclonal tumors might develop over time [11]. Highly heterogeneous tumors exhibit different genotypes among patients or at different sites within a single patient $[12,13]$. Molecular pathology provides directs guidelines for the application of immune checkpoint blockades. However, such examinations have failed to reveal all the molecular information regarding tumor heterogeneity $[14,15]$. Therefore, it is of great significance to reveal the characteristics of various immune cells and the communications between the immune cells and tumor cells as this information would assist in reshaping the tumor microenvironment during antitumor immunotherapy.

F2R is the most extensively studied member of the PAR family $[16,17]$. The 425 amino acids present at the $\mathrm{N}$-terminal domain of F2R are recognized and proteolytically cleaved by specific ligands [18]. The activation of F2R, which depends on the type and the concentration of the acting ligands, then promotes platelet activation, cell proliferation, apoptosis, and angiogenesis [19-21]. GZMA is reported to regulate immune defense by inducing apoptosis, pyrosis, and maintaining homeostasis through the killing of the bacteria and parasites invading the host cells $[22,23]$. Interestingly, GZMA has been reported to competitively interact with F2R against thrombin, although the GZMA-F2R binding has not been demonstrated to induce the coagulation process that is usually induced by the thrombin-F2R interaction [24]. Thus, it is necessary to understand the molecular mechanism underlying the GZMA/F2R communication in cancer patients.

Here, we revealed the following: (i) the quantity and function exhaustion of cytotoxic cells in the tumor tissues of HCC patients; (ii) the failure of the GZMA-F2R communication in tumor tissues; (iii) the induction of caspase3-dependent apoptosis by the

\footnotetext{
${ }^{1}$ Beijing Institute of Hepatology, Capital Medical University, Beijing 100069, China. ${ }^{2}$ Organ Transplantation Center, The Affiliated Hospital of Qingdao University, Qingdao City 266003, China. ${ }^{3}$ Department of Pathology, Inner Mongolia Baogang Hospital, Baotou 014010, China. ${ }^{4}$ School of Chemical, Biological and Environmental Engineering, Carnegie Mellon University, Corvallis, PA 97331, USA. ${ }^{5}$ Department of Clinical Laboratory, Haidian Maternal\&Child Health Hospital, Beijing 100080, China. ${ }^{\circledR}$ email: 1203620677@qq.com Edited by Dr Yufang Shi
}

Received: 10 December 2021 Revised: 4 February 2022 Accepted: 14 February 2022

Published online: 07 March 2022 

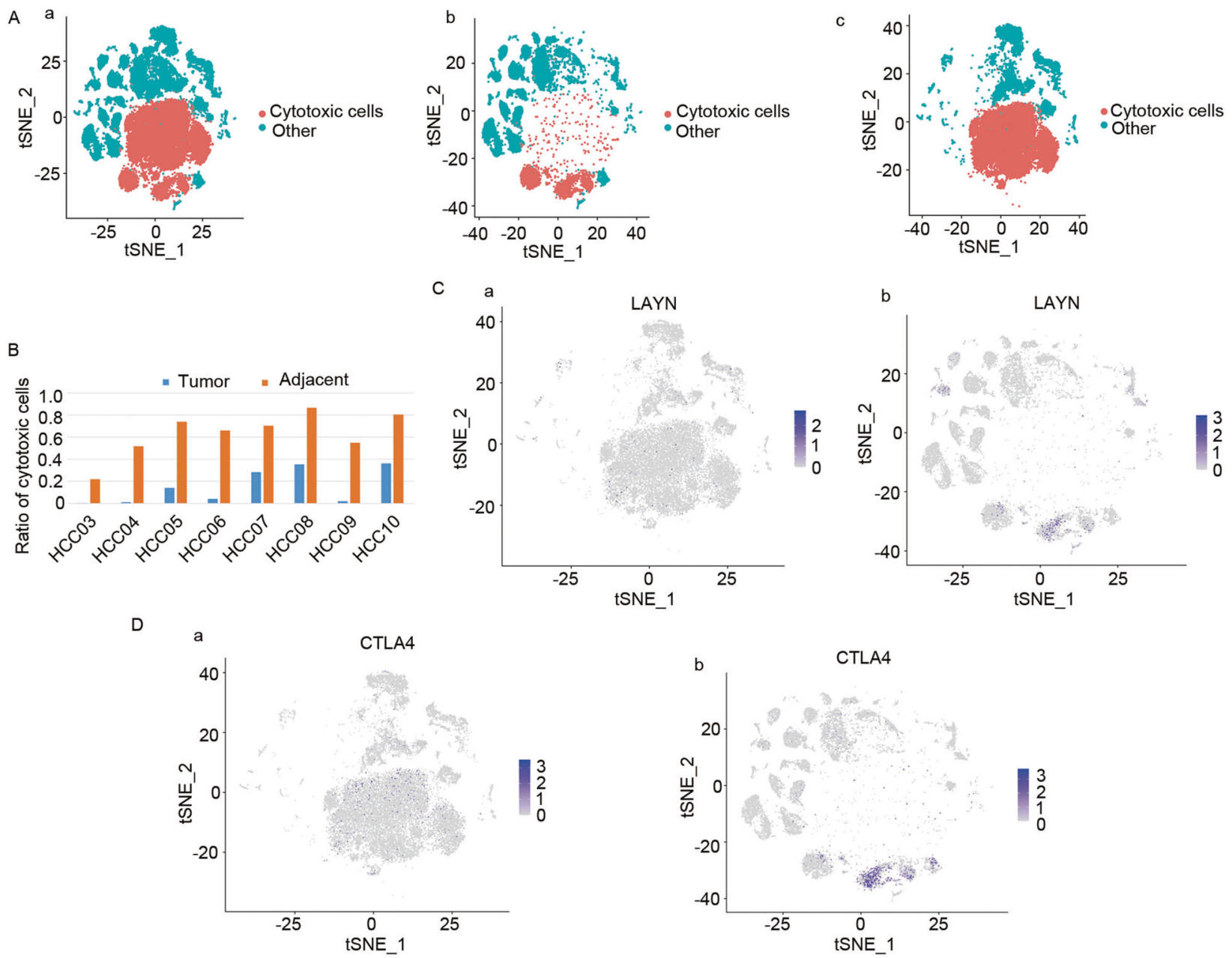

Fig. 1 Single-cell RNA-seq analysis of the cytotoxic cells in the tumor tissue of HCC patients. A The t-SNE map depicting the cytotoxic cells (red) in the (a), tumor tissues (b), adjacent tissues, and (c) tissues from eight HCC patients. B Bar plot depicting the ratio of cytotoxic cells in the tumor tissues and adjacent tissues for each HCC patient. C-D Expression t-SNE maps for the T cell exhaustion markers LAYN and CTLA4 in the tumor tissues and adjacent tissues.

LDPRSFLL motif-activated GZMA-F2R communication via the promotion of the JAK2/STAT1 signaling pathway; (iv) synergistic effect of GZMA-F2R communication and PD-1 mAb therapy in both mouse model and HCC patients; $(v)$ the correlation of the low expression of GZMA/F2R with aggressive clinicopathological characteristics and poor prognosis in HCC patients. The above findings suggested that failure of the GZMA-F2R communication in the tumor tissues of HCC patients limited the antitumor immunotherapy based on immune checkpoint blockades. Therefore, the present study would contribute to and highlighting a completely different direction for scientific research on therapeutic strategy designing in HCC.

\section{RESULTS}

Cytotoxic cell exhaustion in the tumor tissues of HCC patients To well understand HCC, 40195 high-quality, thirty-one subpopulation cells were screened out from a total of 55,632 cells using the following conditions: nFeature_RNA $>500$ \& nCount_RNA $>1000$ \& nCount_RNA $<20000 \&$ percent.mt $<15$ (Fig. S1A). The top five marker genes in subpopulations were visualized on a heatmap (Fig. S1B). The annotate of subpopulations was performed using Human Cell Atlas Data (Table S1). Although a little batch effect was observed (Fig. S1C), the cells from different sources and samples were generally not distributed evenly in subpopulations (Fig. S1D).
These results suggested that the high heterogeneity of HCC patients may be the appropriate reason that generic anti-hepatocellular carcinoma drugs were challenging to be developed [25].

Cytotoxic cells are essential for HCC suppression [26-28]. Unexpectedly, abundant of cytotoxic cells were observed in the adjacent tissues (Fig. $1 \mathrm{Aa}-\mathrm{C}$ and Fig. 1B), while the T-cells exhaustion markers LAYN and CTLA4 were overexpressed in the tumor tissues (Fig. 1C, D). Subsequently, Kyoto Encyclopedia of Genes analysis and Genomes and Gene Ontology analysis were performed for the marker genes of the cytotoxic cells (Table S2). The analyses revealed the enrichment of the apoptotic and T-cell receptor signaling pathways (Fig. S2A). Importantly, in terms of number and function depletion, the gene profile of the cellular components had been reprogrammed, while the molecular function, responses to extracellular factors, and the biological process had been significantly altered (Fig. S2B-E). These results suggested cytotoxic cells exhaustion in the tumor tissues in terms of T-cell receptor and apoptosis activation.

\section{Granzymes failure of cytotoxic cells in the tumor tissue from} HCC patients

Although cytotoxic cell exhaustion in tumor tissues was revealed in the present study, the underlying molecular mechanism was little understood. Here, the cytotoxic cells were isolated (Fig. 2A), and the top 5 marker genes in subpopulation were visualized in a 
A
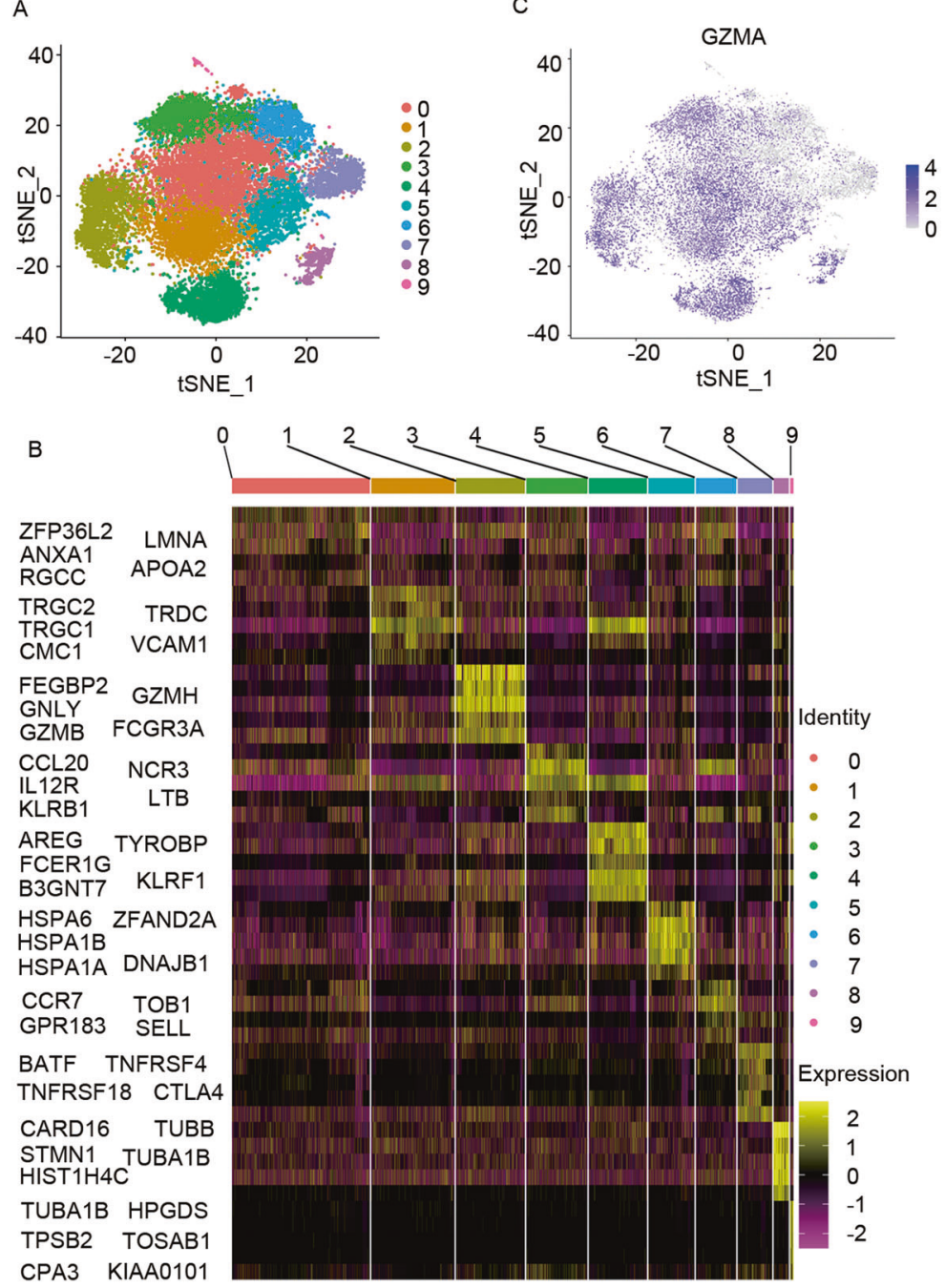

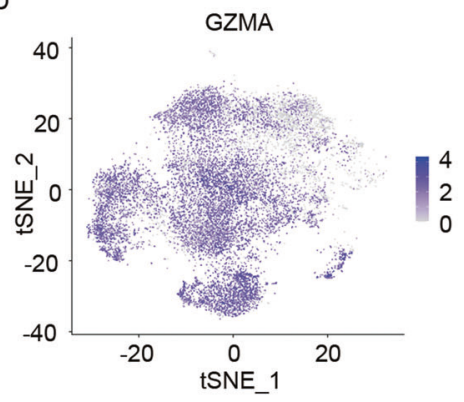

E
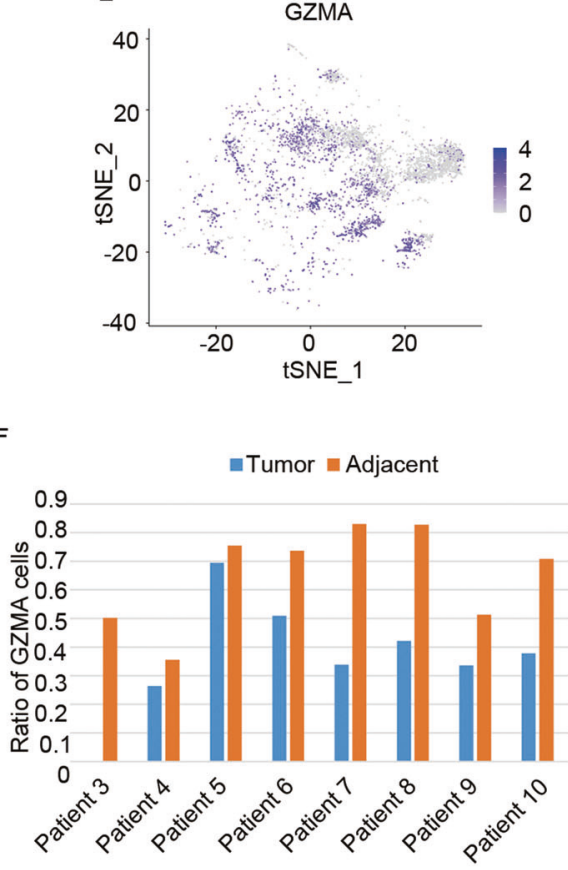

Fig. 2 Single-cell RNA-seq analysis of the granzyme failure in the cytotoxic cells from the tumor tissues of HCC patients. A The t-SNE map depicting clusters of cytotoxic cells in the tumor tissues and adjacent tissues of eight HCC patients. B Expression heatmap of the marker genes in each cytotoxic cell cluster (top 5, color-coded according to the cluster and expression). C Expression t-SNE maps for GZMA in the cytotoxic cells of the tumor tissues and adjacent tissues. D Expression t-SNE maps for GZMA in the cytotoxic cells from the tumor tissues. E Expression t-SNE maps for GZMA in the cytotoxic cells from the adjacent tissues. $\mathbf{F}$ Bar plot illustrating the ratio of GZMA positive cytotoxic cells in the tumor tissues and adjacent tissues for each HCC patient.

heatmap (Fig. 2B). Interestingly, wide expression of granzymes (granzyme $A, B, M, K$, and $H$ ) was detected in all cytotoxic cells (Fig. 2C, Fig. S3Aa, S3Ba, S3Ca, and S3Da). Unfortunately, a massive number of granzyme-negative cytotoxic cells were observed in the tumor tissues (Fig. 2E, Fig. S3Ac, S3Bc, S3Cc, and S3Dc). In addition, the cytotoxic cells and granzyme-positive cytotoxic cells were significantly decreased in the tumor tissues (Fig. 2F, Fig. S3Ad, S3Bd, S3Cd, and S3Dd). According to these results, it was inferred that the granzyme-negative cytotoxic cells in the tumor tissues could be the appropriate reason why the cytotoxic cells failed to lyse the tumor cells.

\section{GZMA-F2R communication failure in the tumor tissues from HCC patients}

In order to further elucidate the mechanism underlying the failure of cytotoxic cells to induce tumor suppression, the cytotoxic cells and tumor cells were separated (Fig. $3 \mathrm{~A}$ ), and subsequently the cell-cell communication was performed using CellChat $\mathrm{R}$ package. Interestingly, fifty-five outgoing communications in five patterns were revealed (Fig. S4A, B). Importantly, the PAR signal secreted by cytotoxic cells was mainly received by the tumor cells (Fig. 3B-D and Table S3), and the GZMA-F2R contributed to the primary PAR communication signaling pathway network (Fig. 3E). Furthermore, GZMA was co-localized with F2R in HepG2 co-cultured with $\mathrm{CD}^{+}$ T-cells and tumor tissues (Fig. 3F). Unfortunately, the GZMApositive cytotoxic cells were detected mainly in the adjacent tissues (Fig. S4C), while the tumor cells from the tumor tissues (cluster 5 and 8) were negative for F2R (Fig. 3G). Together, these results suggested the failure of GZMA-F2R communication in the tumor tissues.

\section{GZMA-F2R communication-mediated tumor cell killing}

The above results demonstrated that there was a failure of GZMAF2R communication in HCC patients. However, the functional role of GZMA-F2R communication remained poorly understood so far in the study. Interestingly, high levels of natural killer cells, activated CD4 $T$ cells, natural killer $T$ cells, and activated CD8 T cells, were observed in high GZMA/F2R expression HCC patients (Fig. 4A). Accordingly, T-cell-mediated tumor cell killing and CCK8 assays were performed in the HepG2 and Huh7 cells infected with F2R-sh-Lv, EGFP-sh-Lv, F2R-Lv, and EGFP-Lv in a co-culture with the $\mathrm{CD}^{+}{ }^{+}$T cells infected with GZMA-rAd, RFP-rAd, GZMA-sh-rAd, 
A
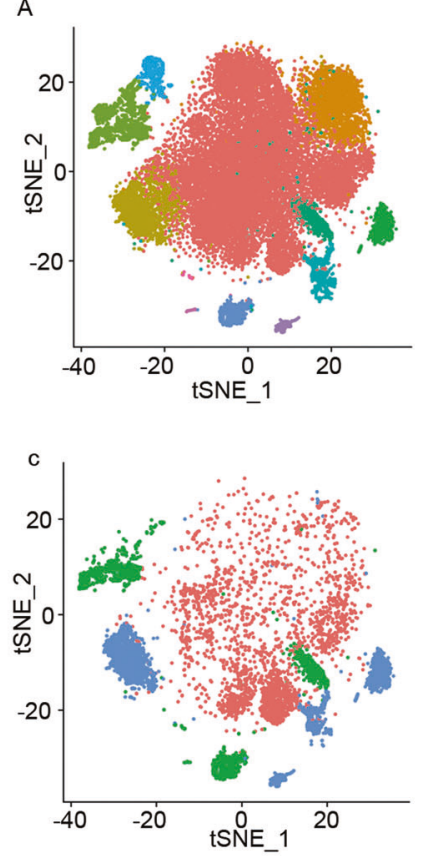

D
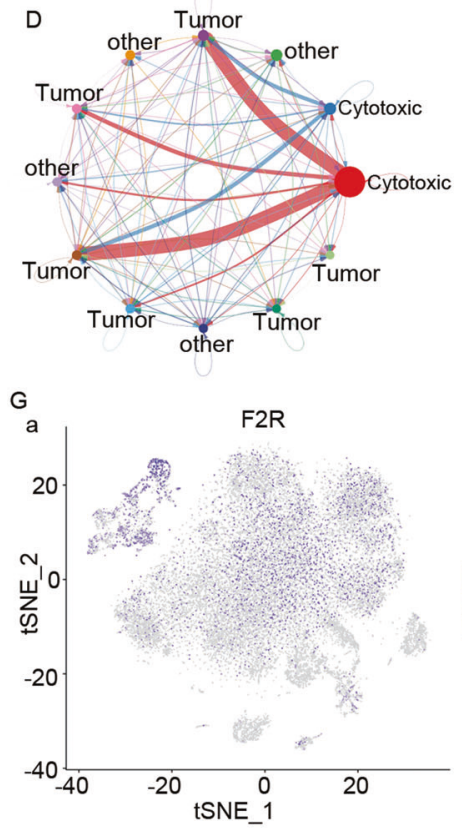

B

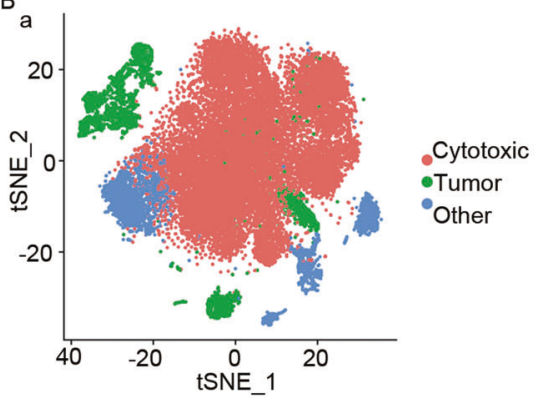

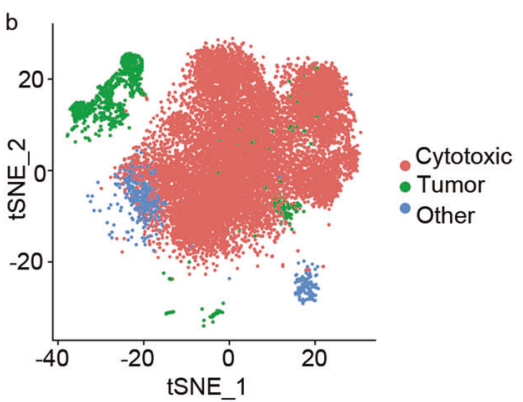

c

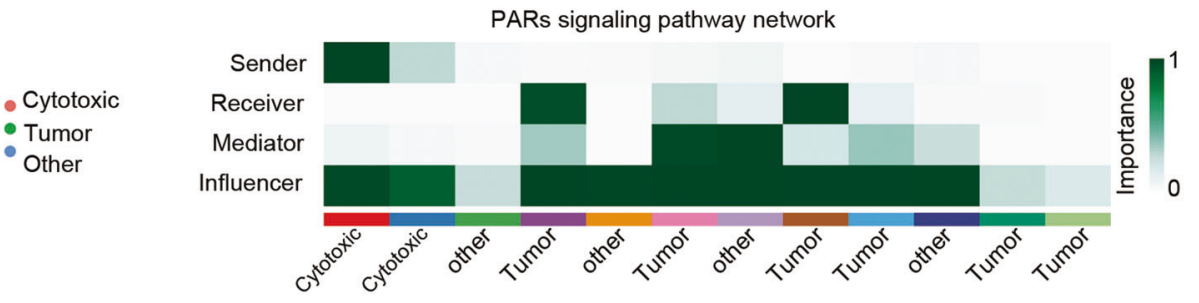

E Contribution of Each L-R Pair

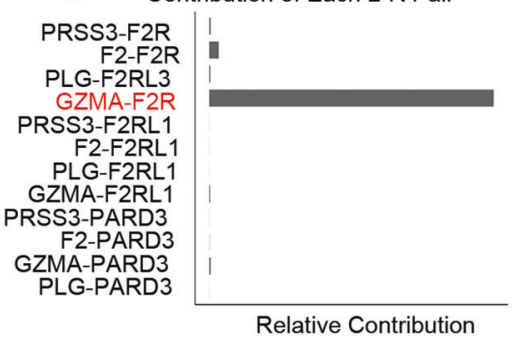

F

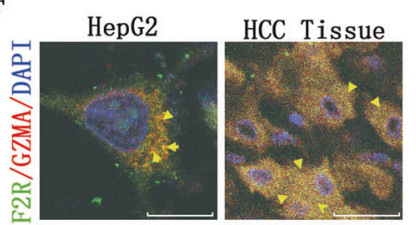

Relative Contribution
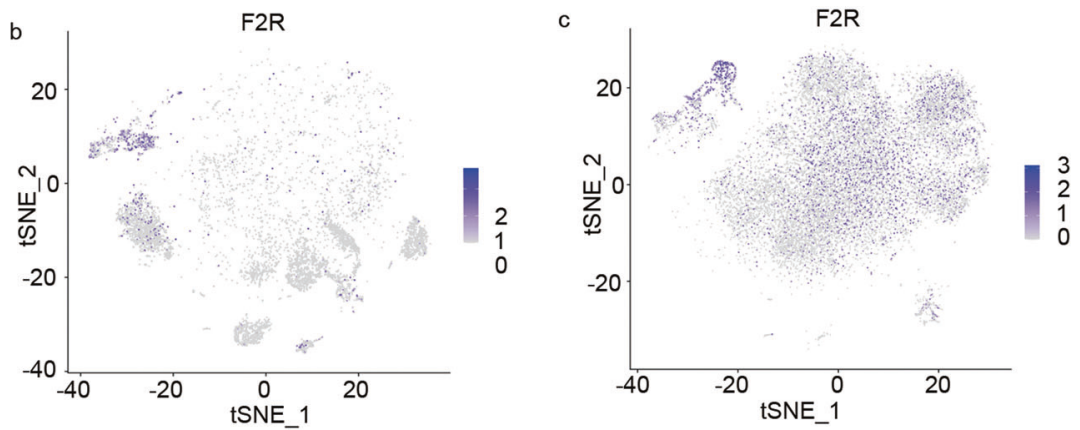

Fig. 3 Cellular communication in cytotoxic cells and tumor cells. A The t-SNE map depicting clusters of cytotoxic cells and tumor cells in the tumor tissues and adjacent tissues of eight HCC patients. B The t-SNE map depicting the types of cytotoxic cells and tumor cells in the tumor and adjacent tissues, tumor tissues, and adjacent tissues of HCC patients, respectively. C Network depicting the PAR signaling pathway in cytotoxic cells and tumor cells. Color indicates the significance of clusters in the PAR signaling pathway. D Circle plot illustrating the PAR signaling pathway in cytotoxic cells and tumor cells. The color and line indicate the significance of clusters in the PARs signaling pathway. E Bar plot illustrating the contribution of ligands and receptors in the PAR signaling pathway in cytotoxic cells and tumor cells. $\mathbf{F}$ Fluorescence confocal assay results demonstrating the co-localization of F2R (green) and GZMA (red) in cultured cells (left) and tissues of an HCC patient (right). The nuclear DNA was stained with DAPI (blue). Scale bars: $15 \mu \mathrm{m}$. G Expression t-SNE maps for F2R in the tumor tissues and adjacent tissues, tumor tissues, and adjacent tissues of eight HCC patients.

and RFP-sh-rAd, respectively. As depicted in Figs. $4 B$ and 4 C, F2R$\mathrm{Lv}$ infection promoted tumor suppression in the $\mathrm{CD}^{+}{ }^{+} \mathrm{T}$-cells infected with GZMA-rAd, while the reverse of this was observed in the cells infected with F2R-sh-Lv and GZMA-sh-rAd.

Induction of apoptosis is an essential function of T cells in tumor suppression [29, 30]. In this context, immunoblotting was performed for activated caspase 3 was evaluation. Lower level of activated caspase 3 were detected in the F2R-sh-Lv-infected Huh7 cells co-cultured with RFP-sh-rAd-infected $\mathrm{CD}^{+}{ }^{+}$cells (Fig. 4Da), while increased levels of activated caspase 3 were observed in the F2R-Lv-infected Huh7 cells cultured with GZMA-rAd-infected CD3 ${ }^{+}$ $T$ cells (Fig. 4Db). Importantly, Calcein AM/PI double staining further confirmed the apoptosis-inducing property of F2R and GZMA expression (Fig. 4E). Together, these results suggested that 

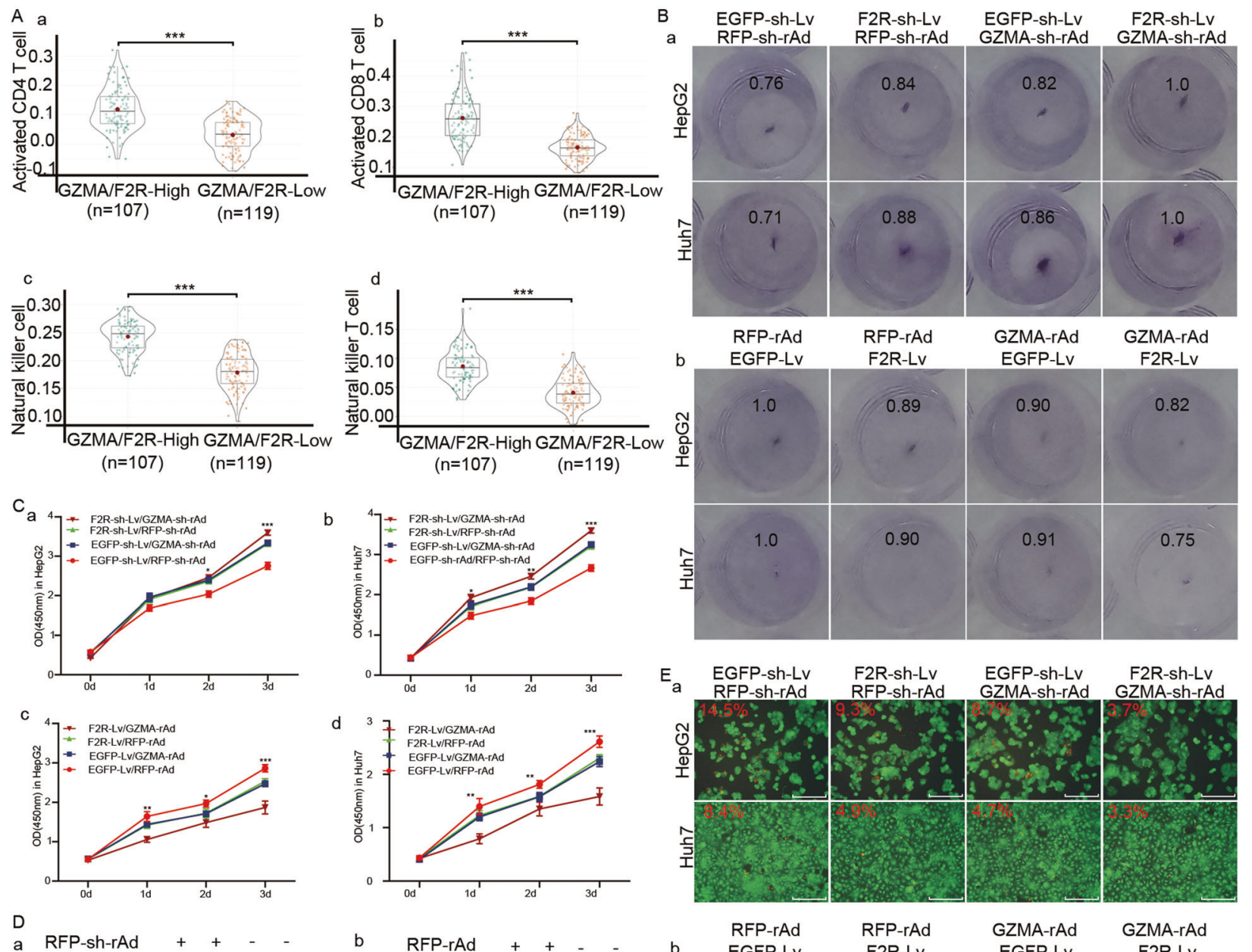
b

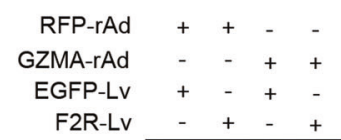
$\begin{array}{rllll}\text { GZMA-sh-rAd } & - & - & + & + \\ \text { EGFP-sh-Lv } & + & - & + & -\end{array}$

F2R-sh-LV

Activated casp3

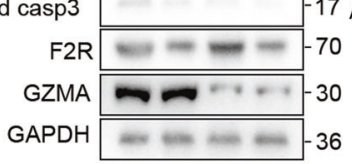

F2R-Lv
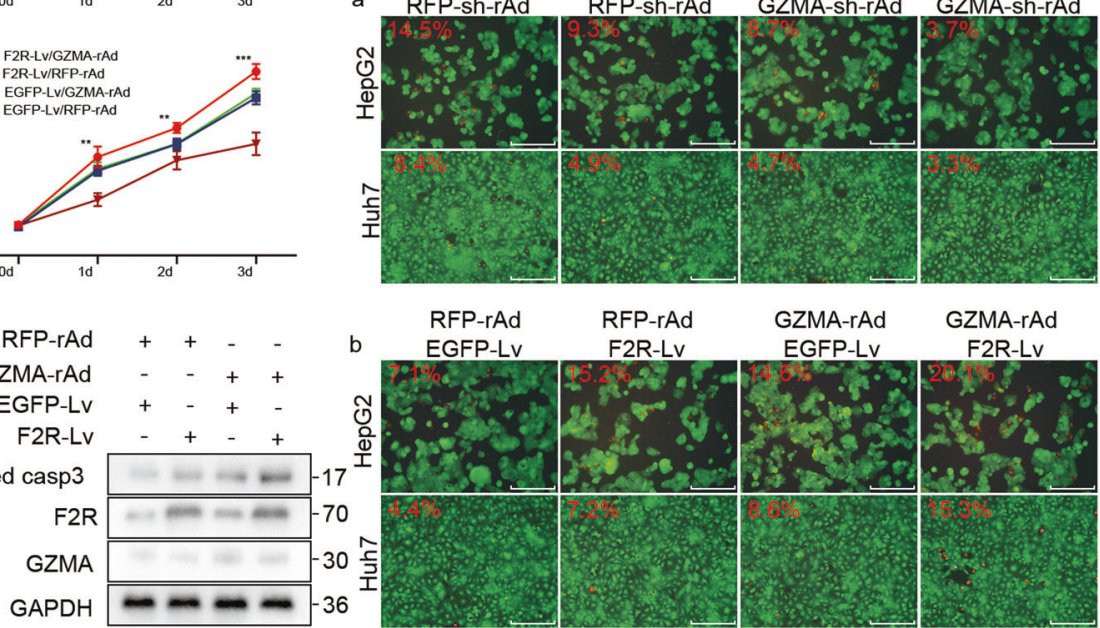

GZMA-rAd

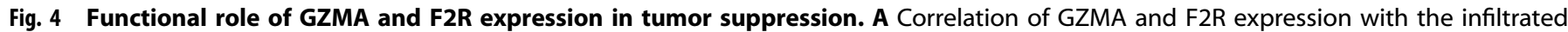

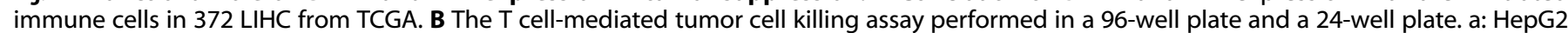

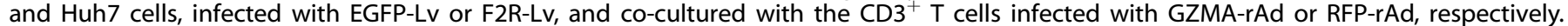

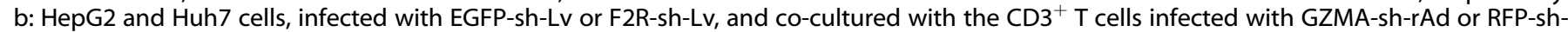

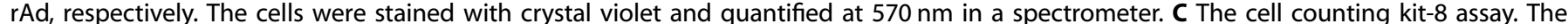

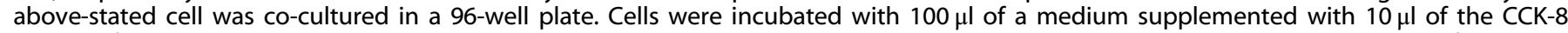

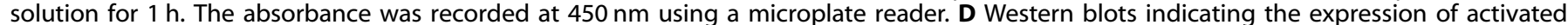

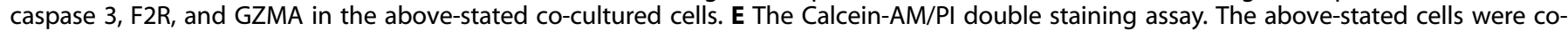

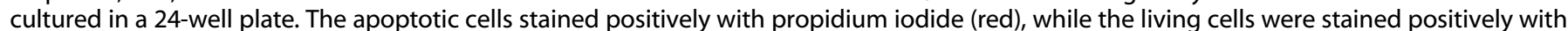

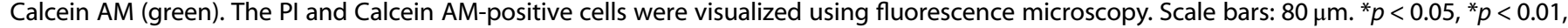
${ }^{* * *} p<0.001,{ }^{* * * *} p<0.0001$.

the GZMA-F2R communication-induced T cell-mediated tumor cell killing that relied on caspase 3 activation.

\section{GZMA-F2R communication promoted JAK2/STAT2 signaling pathway}

In order to decipher the molecular mechanism underlying the GZMA-F2R communication in tumor suppression, the differentially expressed genes in the low F2R/GZMA expression HCC patients were identified (Fig. S5). Moreover, suppression of the IL6/JAK2/ STAT and IL2/STAT signals was revealed in the GSEA analysis (Fig. 5A). Therefore, p-JAK2 was quantified through western blotting in the above cell co-culture system. Interestingly, the levels of p-JAK2 were increased in the F2R-Lv-infected Huh7 cells co-cultured with the GZMA-rAd-infected $\mathrm{CD}^{+} \mathrm{T}$ cells (Fig. 5Bb). On the contrary, the reverse of the above phenomenon was observed in the F2R-sh-Lv-infected Huh7 cells co-cultured with the GZMA-shrAd-infected $\mathrm{CD}^{+} \mathrm{T}$ cells (Fig. 5Ba). Furthermore, western blotting followed by a nuclear extraction revealed the nuclear translocation of STAT1 in the F2R-Lv-infected Huh7 cells co-cultured with the GAMA-rAd-infected $\mathrm{CD}^{+}{ }^{+} \mathrm{T}$ cells, while the reverse of this was observed for the F2R-sh-Lv-infected Huh7 cells co-cultured with the GZMA-sh-rAd-infected T cells (Fig. 5C). The finding of the nuclear 

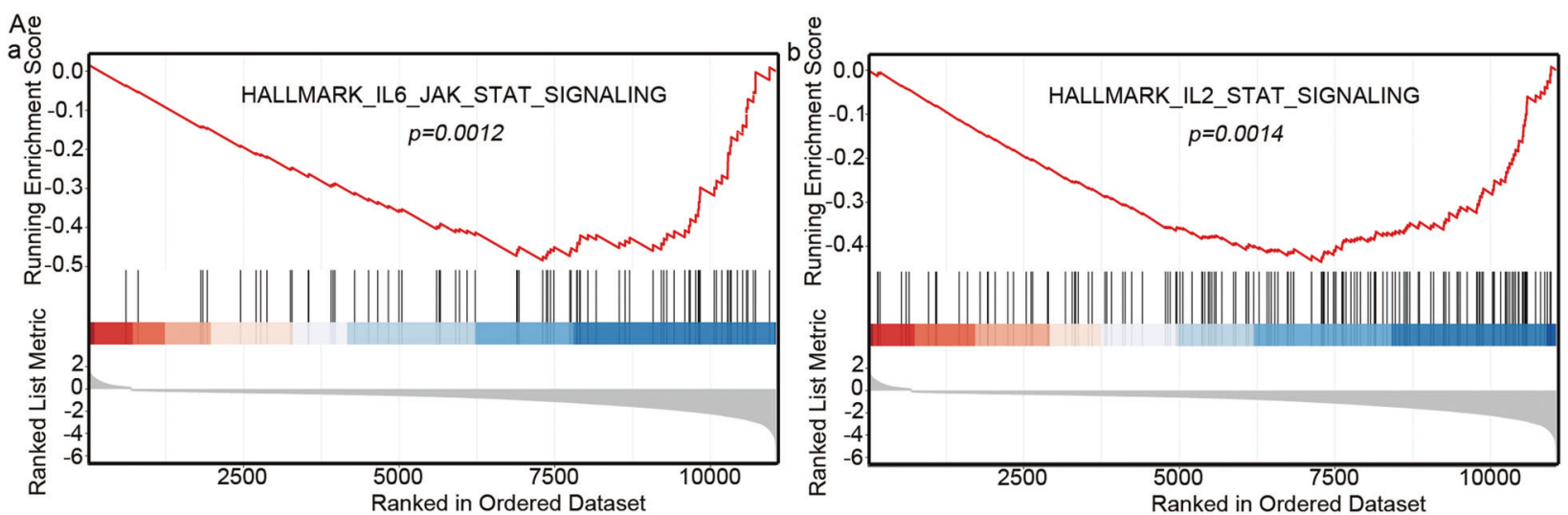

B a
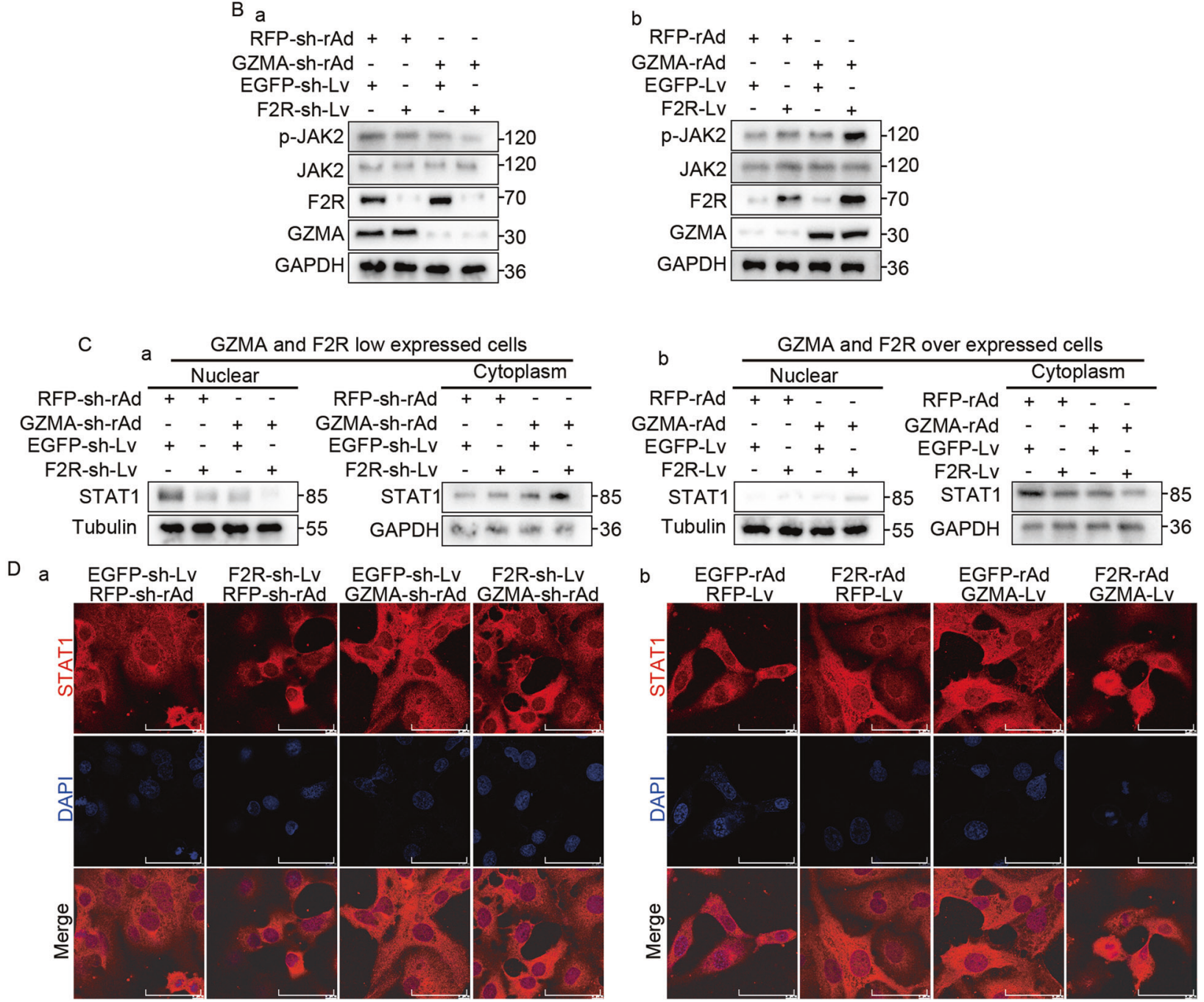

Fig. 5 The molecular mechanism underlying GZMA and F2R expressions in tumor suppression. A Differentially Expressed Genes between high GZMA/F2R expression and low GZMA/F2R expression tissues in the LIHC from TCGA, identified using the gene set enrichment analysis (GSEA). B Western blots indicating the expressions of the JAK2, p-JAK2, F2R, and GZMA in the above-stated co-cultured cells. C Nuclear extraction assay. The nuclear protein and the cytoplasm protein in the above-stated cells were extracted using the nuclear extraction kit. Western blots indicated the level of STAT1 in the nucleus and the cytoplasm. D Fluorescence confocal assay results illustrating the subcellular localization of STAT1 (red) in the above-stated co-cultured cells. The nuclear DNA was stained with DAPI (blue). Scale bars: $15 \mu \mathrm{m}$.

translocation was further confirmed in the results of the immunofluorescence assay (Fig. 5D). Together, these results suggested that the GZMA-F2R communication suppressed the tumor by promoting the activation of JAK2/STAT1 signaling pathway.
LDPRSFLL motif in the F2R-activated GZMA-F2R communication In order to completely understand the mechanism underlying the GZMA-F2R communication, LDPRSFLL-deleted and LDPRSFLLmutated motifs (Fig. S6) were generated using PCR. As depicted 
in Fig. 6A, B, increased levels of activated caspase 3 and p-JAK2 were detected in the F2R-rAd-infected Huh7 cells co-cultured with $\mathrm{CD}^{+} \mathrm{T}$ cells, and these levels were decreased in the F2R-rAdinfected cells containing the LDPRSFLL-mutated or LDPRSFLLdeleted motifs and the cells incubated with the LDPRSFLL motifspecific inhibitors SCH530348 and SCH79797. Importantly, the T cell-mediated tumor cell killing assay and Calcein-AM/PI double staining confirmed the promotion of F2R-induced tumor suppression by the LDPRSFLL motif (Fig. 6C, D). Consistently, western blotting followed by a nuclear extraction revealed the nuclear translocation of STAT1 in the F2R-rAd-infected Huh7 cells cocultured with $\mathrm{CD}^{+}{ }^{+}$cells, while the reverse of this was observed in the F2R-rAd-infected cells containing the LDPRSFLL-mutated or LDPRSFLL-deleted motif and the cells incubated with SCH530348 or SCH79797 (Fig. 6E). The nuclear translocation of STAT1 was further confirmed in the immunofluorescence assay (Fig. 6F). Together, these results demonstrated that the GZMA-F2R communication-induced tumor suppression might be promoted by the LDPRSFLL motif.

\section{Low expression of GZMA and F2R impair the therapeutic} efficacy of PD-1 mAb in both mouse model and HCC patients The above result demonstrated that the GZMA-F2R communication promotes cytotoxicity in tumor suppression. It is widely accepted that PD-1 and PD-L1 expressions impair antitumor therapy [31, 32]. In this context, the relationship among GZMA, F2R, PD-1, and PD-L1 in TCGA, LIHC, and GTEx was analyzed in the present study. The results revealed that GZMA and F2R were positively correlated with PD-1 and PD-L1 in cancers and tissues (Fig. S7A-S7C). Therefore, to fund out the role of F2R in the tumor suppression of PD-1 mAb therapy, Hepa1-6 cell, $5^{*} 10^{5}$, infected with F2R-Lv or EGFP-Lv, were subcutaneously injected into immune-competent $\mathrm{C} 57 \mathrm{BL} / 6$ mice that had been previously injected with PD-1 mAb or lgG2a (Fig. 7A). Interestingly, decreased tumor growth (Fig. 7B and Fig. S8A) and increased levels of activated caspase 3 and apoptotic cells (Fig. S9A) were observed in the F2R-Lv-infected and PD-1 mAb-injected mice.

In order to further understand the role of the GZMA-F2R communication in the F2R-promoting effects of PD-1 mAb in tumor suppression, $5 * 10^{5}$ Huh7 cells-infected with F2R-sh-Lv or EGFP-sh-Lv were subcutaneously injected into immune-deficient $B A L B / C$ nude mice. Subsequently, $1 * 10^{6} \mathrm{CD}^{+} \mathrm{T}$ cells-infected with GZMA-sh-rAd or RFP-sh-rAd and PD-1 mAb were injected into the mice to rebuild the immune system (Fig. 7C). Increased tumor growth (Fig. 7C and Fig. S8B) and decreased levels of activated caspase 3 and apoptotic cells (Fig. S9B) were observed in the F2Rsh-Lv and GZMA-sh-rAd-infected and IgG mAb treated mice. Unfortunately, no significant change in tumor growth was observed in the F2R-sh-Lv-infected mice. Interestingly, increased tumor growth and decreased levels of caspase 3 activation and apoptosis cells were observed in GZMA-sh-rAd infected mice (Fig. 7C, D and Fig. S8B and S9B). These results demonstrated that low expression of F2R and GZMA resulted in poor ability of PD-1 $\mathrm{mAb}$ for tumor suppression.

In order to confirm the above findings in cancer patients, 18 PD-L1-positive HCC patients injected with PD-1 mAb, comprising ten responders, and eight non-responders (Table S4), were recruited for the analysis of GZMA and F2R expressions. Two representative cases of tumor diameter change (indicated with a red line) in response to PD- 1 mAb therapy were are depicted in Fig. 7E, while two representative cases of GZMA and F2R are depicted in Fig. 7F. Importantly, low expressions of GZMA and F2R were observed in the PD- 1 mAb non-responder patients (Fig. 7G). In addition, the expressions of GZMA and F2R were positively correlated with the changed diameter (Figs. $7 \mathrm{H}, \mathrm{I}$ ). Together, these results suggested that the tumor suppression property of PD-1 mAb was impaired in the case of GZMA-F2R communication failure.
Downregulation of GZMA and F2R was associated with aggressive clinicopathological characteristics and a poor prognosis in HCC patients

In order to better understand the role of GZMA-F2R communication in HCC, the GZMA and F2R RNA-seq data from 33 cancer types and nine $\mathrm{HCC}$ datasets were analyzed. The analysis revealed a downregulation of F2R in the tumor tissues of CESC, KICH, KIRP, LUAD, LUSC, and UCEC, while a low expression of GZMA was observed in COAD, LUAD, LUSC, UCEC, PAAD, and READ (Fig. S10A). In the HCC RNA-seq data from GEO, F2R expression was observed to be significantly downregulated in 5 datasets, while the GZMA expression was significantly downregulated in all datasets (Fig. S10B). Consistent with this result, the downregulation F2R and GZMA in the tumor tissue of HCC patients was also observed in the western blotting, quantitative real-time polymerase chain reaction (Fig. 8A, B; Table S5 and S6), and tissue microarray (Fig. $8 \mathrm{C}$ ) analyses. These results confirmed that F2R and GZMA were downregulated in the tumor tissues.

In order to completely understand the contribution of F2R and GZMA, the correlation of the downregulation of F2R and GZMA with sex, vascular infiltration, and encapsulation was evaluated (Fig. S11A). The low expression of F2R/GZMA was observed to be correlated with poor overall survival (median OS times:526 vs. 673 days; $P=0.034$ ), shorter time to disease-free survival (median DSS times: 526 vs. 673 days; $P=0.016$ ), and worse progressionfree survival (median PFS times:363 vs. 524 days; $P=0.022$ ) (Fig. $\mathrm{S} 10 \mathrm{C}$ ). Similarly, low expression of the F2R/GZMA protein was correlated with poor overall survival (median OS times:27.35 vs. 49.25 months; $P=0.027$ ) and shorter time to recurrence (median TTR times: 31.15 vs. 43.00 months; $P=0.015$ ) in HCC patients (Fig. $8 D$ ). Furthermore, the multivariate analysis revealed that $F 2 R$ and GZMA, together with microvascular invasion, and AFP, are independent risk factors for both OS and TTR (Fig. S11B). The low F2R-expression patients exhibited a higher risk for tumor recurrence (TTR: $H R=0.974 ; 95 \%$ confidence interval: 0.951-0.997; $P=0.029$ ), while the low GZMA expression patients exhibited a shorter OS rate and a higher risk for tumor recurrence $(\mathrm{OS}: \mathrm{HR}=$ 0.973; 95\% confidential interval: 0.955-0.992; $P=0.007$; TR: HR $=$ 0.973; 95\% confidence interval: $0.955-0.991 ; P=0.004)$. According to these, it was inferred that the frequently downregulated F2R and GZMA correlated with aggressive clinicopathological characteristics and a poor prognosis in HCC.

\section{DISCUSSION}

Tumor progression is closely associated with the antitumor activity of the immune system [33]. In tumors, T cells exhaustion in terms of highly expressed immunosuppressive receptors and reduced secretion of functional cytokines is reported [34], which induces $T$ cell inactivation and inefficiency during the antitumor process, thereby resulting in immune escape $[35,36]$. In the present study, it was revealed that the cytotoxic cells in tumor tissues are simultaneously exhausted in both quantity and function in terms of activation of TCR and apoptotic signals (Fig. 1B, C and Fig. S2A-2B). Meanwhile, a decrease in the secreted granzyme and the proportion of granzyme positive cells was observed in the tumor tissues (Fig. 2 and Fig. S3). Therefore, a widely accepted essential strategy for tumor immunotherapy is to reverse the immune response of exhaustive $T$ cells via the blocking of immune checkpoints [37].

In practice, antitumor therapy uses PD-1 mAb to neutralize the immunosuppressive receptor PD-1 [38], while PD-L1 mAb is used for neutralizing PD-L1 [39, 40]. Unfortunately, an increased number of patients failed to exhibit a long-term response to PD$1 \mathrm{mAb}$ or PD-L1 mAb immunotherapy for heterogeneous immune and tumor cells [41]. In the present study, the GZMA-F2R communication promoted JAK2/STAT1 signal-induced tumor suppression both in vitro and in vivo (Figs. 4, 5, and 7). 

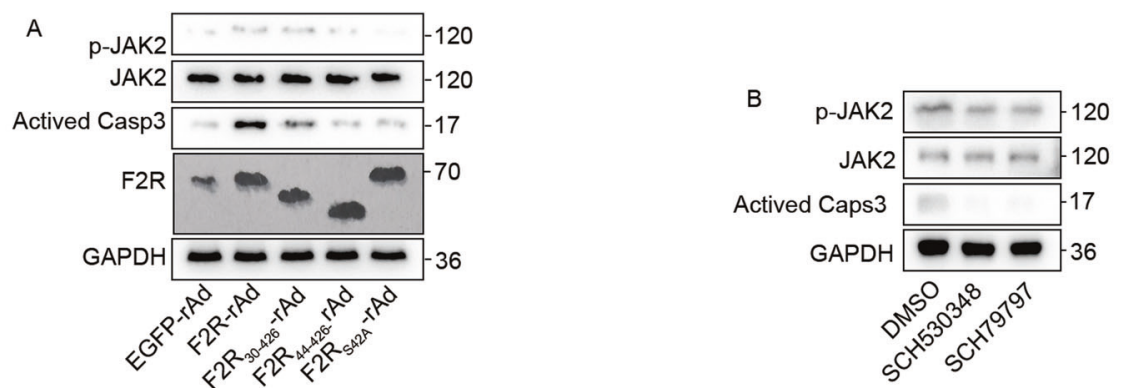

C
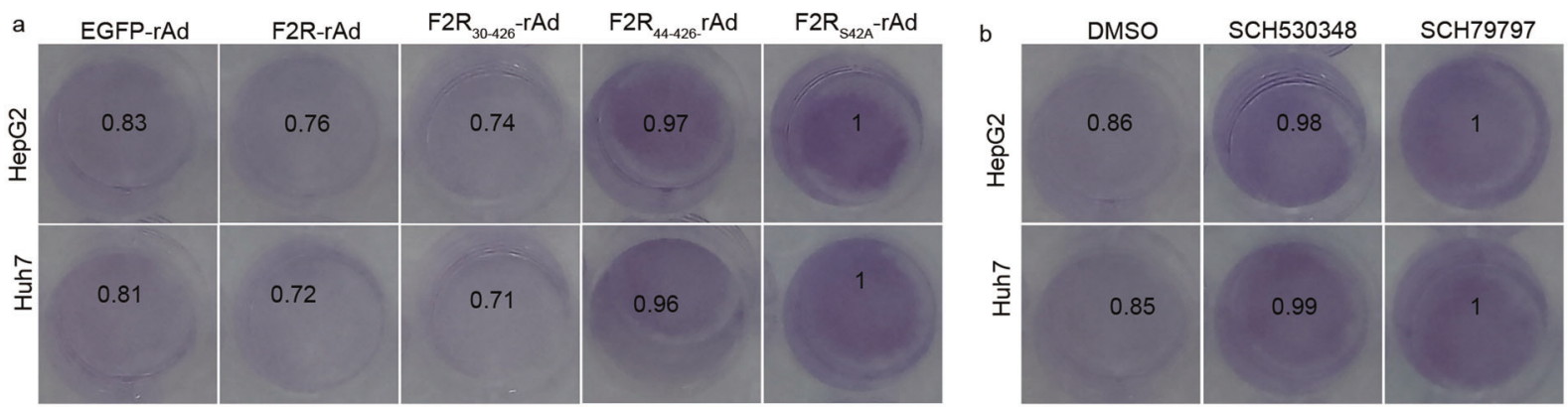

D
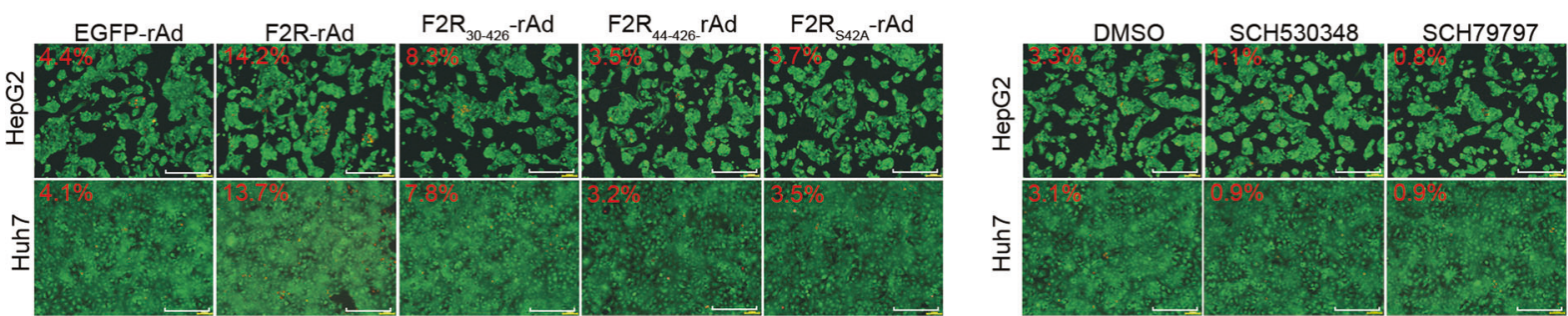

E
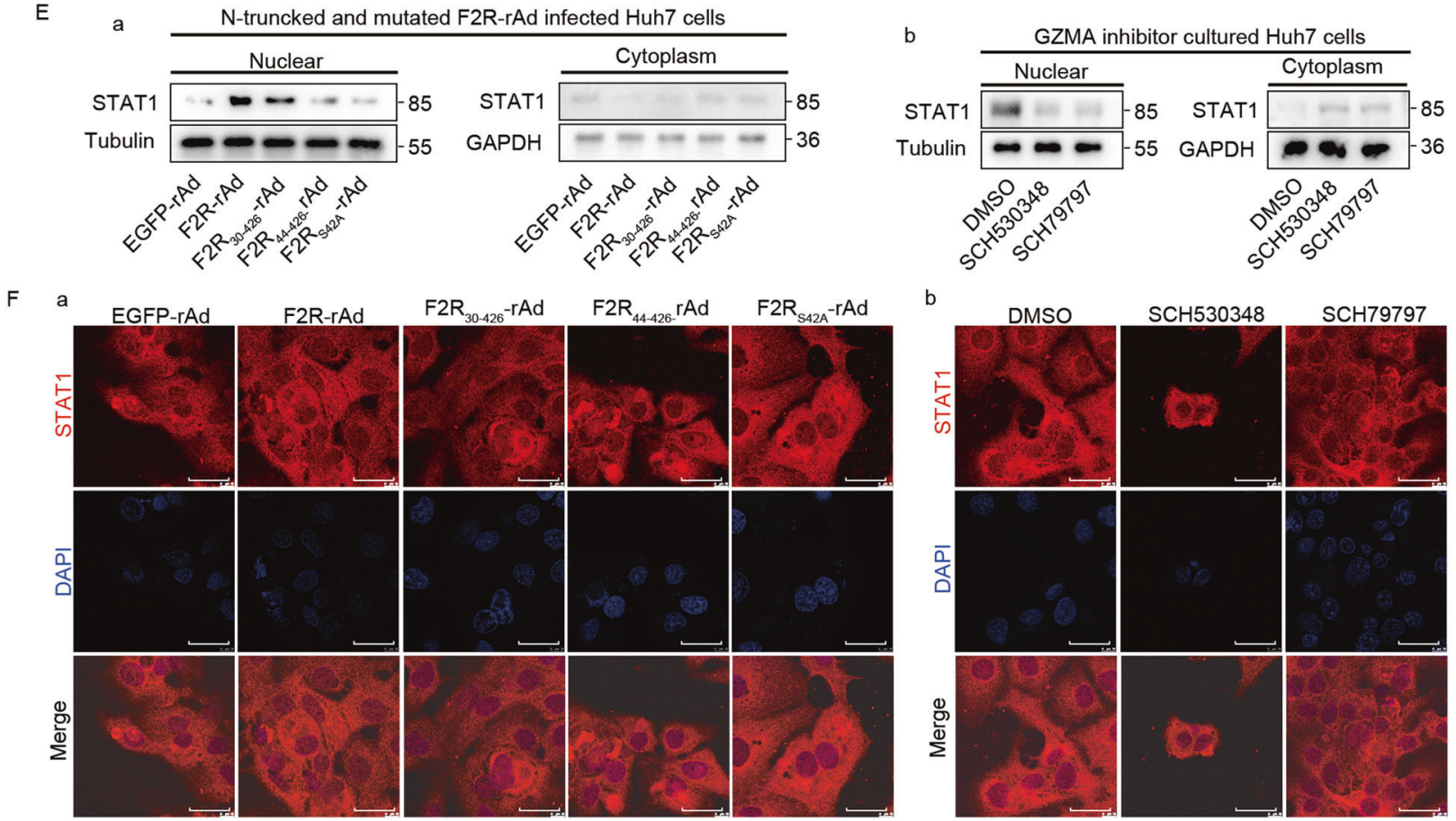

Unfortunately, two tumor cells exhibited negative expression of F2R in tumor tissues (Fig. 3D). Moreover, the low expression of GZMA and F2R was positively correlated with PD-1 and PD-L1 (Fig. S7), impaired tumor suppression by PD-1 mAb (Fig. 7, Fig. S8, Fig. 9), and also predicted aggressive clinicopathological characteristics and a poor prognosis (Fig. 8, Fig. S10, and Fig. S11). Therefore, the therapy strategies based on GZMA-F2R communication would be extremely potent in reversing the inefficient antitumor immunotherapy based on PD-1 mAb and PD-L1 mAb. 
Fig. 6 The molecular mechanism underlying the role of F2R in GZMA expression-based tumor suppression. A Western blots indicating the expressions of activated caspase3, JAK2, p-JAK2, and F2R in the CD3 ${ }^{+}$cells co-cultured with the Huh7 cells infected with F2R-rAd, LDPRSFLL mutated motif F2R-rAd, and LDPRSFLL deleted motif F2R-rAd. B Western blots indicating the expressions of activated caspase3, JAK2, p-JAK2, and $\mathrm{F} 2 \mathrm{R}$ in the $\mathrm{CD}^{+}$cells co-cultured with the Huh7 cells incubated with the LDPRSFLL motif-specific inhibitor (either SCH530348 or SCH79797). C The T cell-mediated tumor cell killing assay. The above-stated cells were stained with crystal violet and quantified at $570 \mathrm{~nm}$ in a spectrometer. D The Calcein-AM/PI double staining assay. The above-stated cells were co-cultured in 24-well plates. The apoptotic cells stained positively with propidium iodide (red), while the living cells stained positively with Calcein AM (green). The PI and Calcein AM-positive cells were visualized using fluorescence microscopy. Scale bars: $80 \mu \mathrm{m}$. E The nuclear extraction assay. The nuclear protein and the cytoplasmic protein in the above-stated cells were extracted using the nuclear extraction kit. Western blots indicated the level of STAT1 in the nucleus and the cytoplasm. F Fluorescence confocal assay results illustrating the subcellular localization of STAT1 (red) in the above-stated co-cultured cells. The nuclear DNA was stained with DAPI (blue). Scale bars: $15 \mu \mathrm{m}$.

However, the present study revealed that the expressions of GZMA and F2R were positively correlated with PD-1 and PD-L1 (Fig. S7) and regulated the tumor suppression in PD-1 mAb therapy in both mouse models and HCC patients (Fig. 7). Moreover, the molecular mechanism underlying the GZMA-F2R communication in antitumor immunotherapy and its role in PD-1suppressed tumor progression were elucidated (Figs. 3-8). However, to elucidate the specific molecular mechanisms underlying the expressions of PD-1 and PD-L1 in the GZMA-F2R communication failure, further investigation is warranted.

Thrombin is the most extensively elucidated ligand that interacts with F2R and promotes tumor progression [42-44]. However, multiple principal protease cleavage sites, thrombin, cathepsin, proteinase3, and human leukocyte elastase have been characterized at the N-terminal of F2R [24, 45]. In addition, F2R is reported to perform its biological functions in a ligand and concentration-dependent manner [46]. However, GZMA and thrombin recognize the same domain, named LDPRSFLL, although the interaction of thrombin with F2R induces platelet aggregation while the interaction of GZMA with F2R fails to induce platelet aggregation [47]. In the present study, the GZMA-F2R communication at the LDPRSFLL motif of F2R suppressed tumor progression by promoting the JAK2/STAT1 signal activationinduced apoptosis (Figs. 4-6). Interestingly, tumor volume was promoted in low GZMA expression mice while such promotion did not occur in the low F2R expression mice (Fig. 7 and Fig. S8). These results implicated that GMZA might be suppressing tumors via F2R and another factor. Assuredly, perforin delivers GZMA to the cytosol of target cell and suppresses tumor progression in caspase-independent apoptosis [48, 49].

The failure of the GZMA-F2R communication was identified in the tumor tissues of HCC patients. It was revealed that the binding of GZMA to the LDPRSFLL motif at the N-terminus of F2R promotes apoptosis via JAK2/STAT1 signaling, which in synergy with the PD-1 mAb therapy led to tumor suppression in both mouse model and HCC patients (Fig. S12). Therefore, a combination therapy comprising the modulation of GZMA-F2R communication and the use of an anti-PD-1 antibody would exhibit much better antitumor efficacy in the treatment of HCC patients.

\section{MATERIALS AND METHODS \\ Data collection}

Single-cell sequencing matrices (GSE149614) and nine RNA-Seq datasets (GSE14520, GSE36376, GSE46444, GSE54236, GSE57957, GSE64041, GSE76297, GSE10207, and GSE121248) were download directly from the GEO. The RNA-seq data for 33 types of tumors were downloaded from the Genomic Data Commons Data Portal.

\section{Patients and animals}

A total of 158 pairs of tumor and adjacent tissue from HCC patients, for use in tissue microarray staining and total RNA or protein isolation, were collected from the Affiliated Hospital of Qingdao University, between March 2014 and August 2017 (Table S6). In addition, paraffin sections of 18 HCC patients who underwent PD-1 mAb treatment at the Affiliated
Hospital of Qingdao University, between May 2017 and November 2020 (Table S4) were collected. Peripheral blood for PBMCs and T cell isolation was collected from healthy donors. The patients and their families were thoroughly informed regarding the study, and their approval was obtained in a written informed consent form that was signed by each participant. The present study was conducted under the guidelines and principles of the Declaration of Helsinki.

Six-week-old male $C 57 B L / 6$ or $B A L B / c$ nude mice were procured from SPF (Beijing) Biotechnology Co. Ltd. The BALB/C nude mice were then randomly distributed in individually ventilated cage (IVC) systems, while the C57BL/6 mice were housed in specific pathogen-free (SPF) grade animal rooms. All animal experiments were conducted under guidelines of the Animal Care Facility of Qingdao University and those of the National Institute of Health. The study protocols were approved by the ethics committee of the Affiliated Hospital of Qingdao University (QYFYWZLL26539).

\section{Cell culture}

HepG2, Huh7, Hepa1-6, and HEK-293A cell lines were purchased and authorized (STR profiling) from the China Center for Type Culture Collection. The cell lines were cultured in DMEM or MEM supplemented with $10 \%$ fetal bovine, $100 \mu \mathrm{g} / \mathrm{mL}$ streptomycin, and $100 \mathrm{U} / \mathrm{mL}$ penicillin. T cells were cultured in the RPMI-1640 supplemented with Recombinant Human IL-2, $10 \%$ fetal bovine, $100 \mu \mathrm{g} / \mathrm{mL}$ streptomycin, and $100 \mathrm{U} / \mathrm{mL}$ penicillin.

\section{Separation of PBMCs}

Peripheral blood mononuclear cells (PBMCs) were separated as described in a previous report [50]. Briefly, peripheral blood was collected in a $15-\mathrm{ml}$ tube and centrifuged at $2000 \mathrm{rpm}$. The cell pellet was collected diluted, and mixed gently with 1x PBS. Subsequently, the lymphocyte separation medium was added to the PBMCs separation tube, followed by the addition of blood cells on the top of the lymphocyte separation medium. After $10 \mathrm{~min}$ of centrifugation at $2000 \mathrm{rpm}$, the separated cells were collected and washed twice with 1x PBS. Subsequently, the red blood cell lysis buffer was added to lyse the red blood cells, followed by two washes with 1x PBS.

\section{Separation of $\mathrm{CD}^{+} \mathrm{T}$ cell}

The $\mathrm{CD}^{+}{ }^{\mathrm{T}}$ cells were separated as described in a previous report [51]. The $\mathrm{CD} 3 / \mathrm{CD} 28$ conjugated magnetic beads were vortexed, and then, $100 \mu \mathrm{l}$ of the bead mixture was transferred to an EP tube and resuspended in PBS, while the supernatant was discarded. The resuspended beads were then mixed with the separated cells and incubated inside a shaker incubator for $30 \mathrm{~min}$. Afterward, the mixture was equilibrated on a magnetic stand for 2 min. Finally, the mixture of cells and beads was resuspended and cultured for 9-14 days.

\section{Construction of vectors}

Adenovirus was produced via the double transfection of HEK-293A cells with the adenoviral backbone vector (pDC316-GZMA-shRNA for the construction of shRNA-GZMA and pDC316-mCMV-EGFP for the construction of F2R-rAd, F2R $30-426-r A d, F 2 R_{44-426}-r A d$, and F2R $\left.R_{542 A}-r A d\right)$ and the packaging plasmid pBHGlox-E1,3Cre in a ratio of 1:1 using Lipofectamine 3 000 (Invitrogen, CA, USA). A lentiviral transfer vector (pLV-CMV-shRNA for the construction of shRNA-F2R and pLV-F2R vector for construction of $L v$ $\mathrm{F} 2 \mathrm{R}$ ) and two packaging plasmids $\mathrm{pH} 1$ and $\mathrm{pH} 2$ were triple transfected in a ratio of 0.5:0.35:0.15 into the HEK-293T cells for Lentivirus construction. The target sequences used for F2R and GZMA gene interference are provided in Table S5. 
A

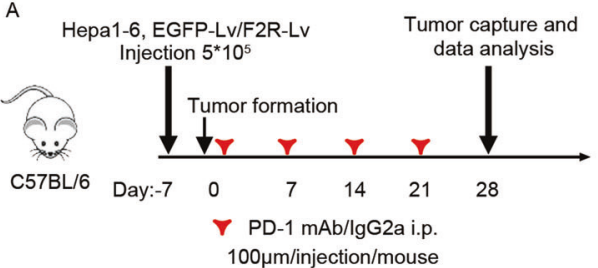

c

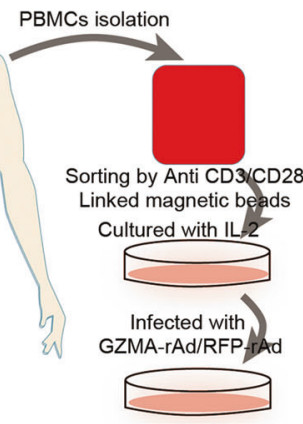

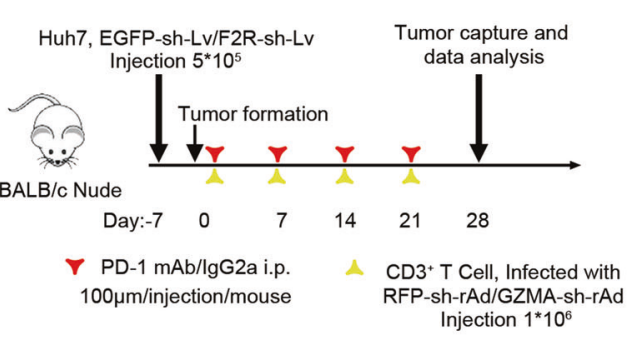

$E$

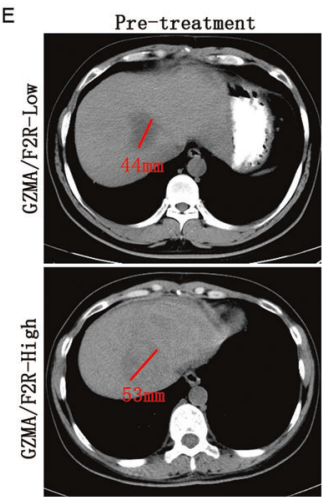

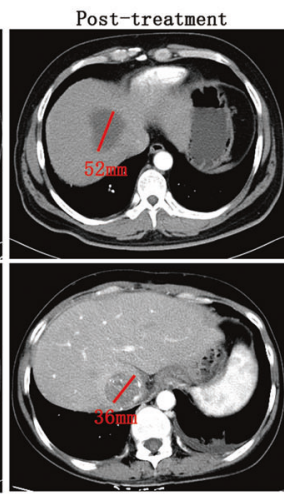

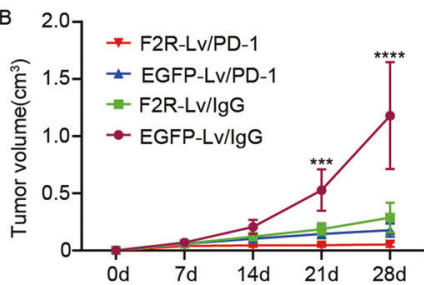

-i- RFP-sh-rAd/EGFP-sh-Lv/PD-1

- RFP-sh-rAd/EGFP-sh-Lv/lgG

RFP-sh-rAd/F2R-sh-Lv/PD-1

$\rightarrow$ GZMA-sh-rAd/EGFP-sh-Lv/PD-1

- GZMA-sh-rAd/EGFP-sh-Lv/lgG

- GZMA-sh-rAd/F2R-sh-Lv/PD-1

$\rightarrow$ GZMA-sh-rAd/F2R-sh-Lv/lgG
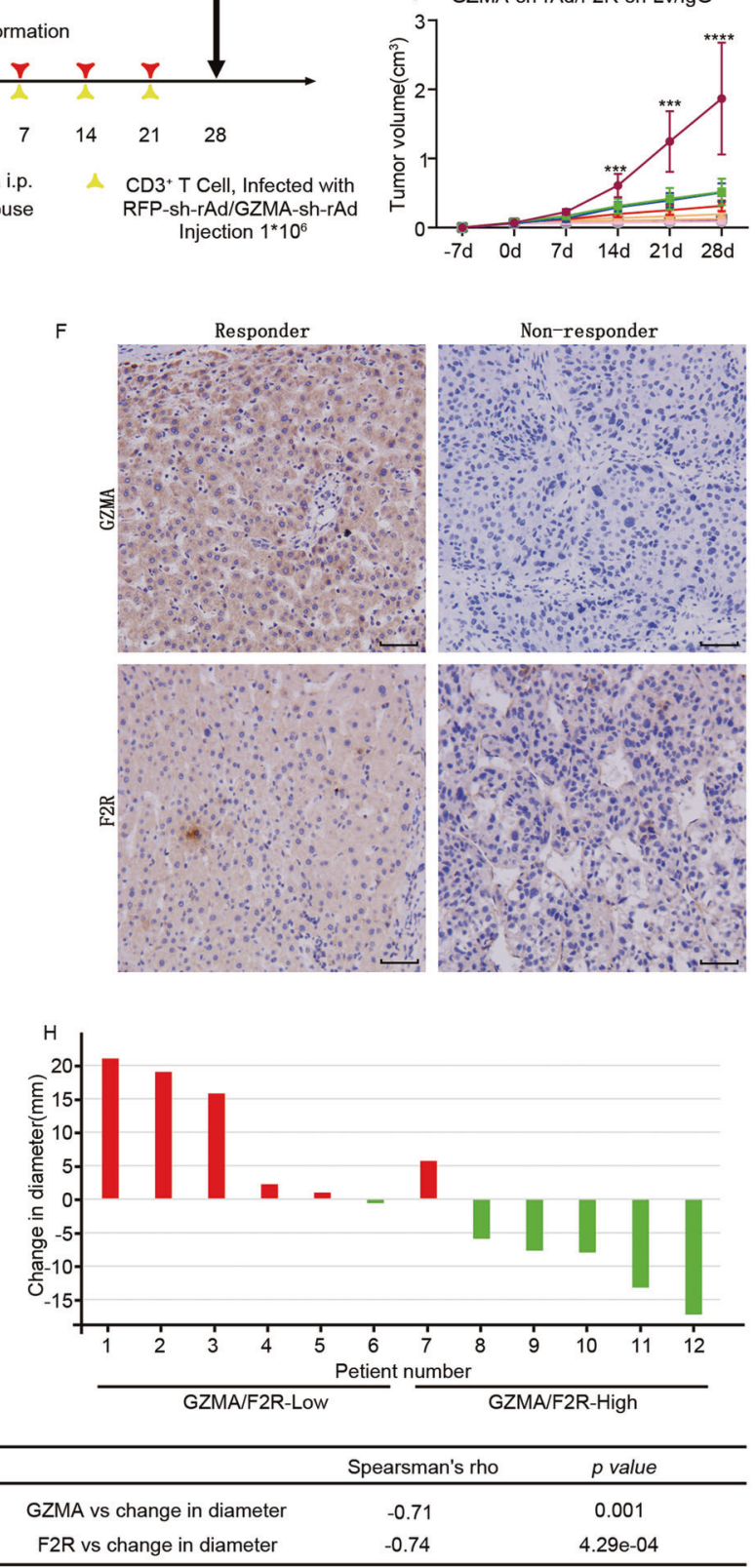

\section{In vivo tumor model}

In order to establish the immune-competent mouse model, $4 \times 10^{6} \mathrm{Hepa1-}$ 6 cells were mixed with Matrigel and then inoculated into C57BL/6 mice for the establishment of an allograft HCC mouse model. In order to establish the immunodeficient mouse model, $2 \times 10^{6}$ Huh7 cells were inoculated into $\mathrm{BABL} / \mathrm{C}$ nude mice for developing the xenograft HCC mouse model. $\mathrm{CD}^{+} \mathrm{T}$ cells were injected into the mice via the tail vein to reconstitute the human immune system. PD1 mAb (Sintilimab, $10 \mu \mathrm{g} / \mathrm{kg}$ ) or an IgG was 
Fig. 7 Functional role of GZMA and F2R expressions in the antitumor efficacy of PD-1 mAb therapy. A, B The C57BL/6 mice were injected with $5 \times 10^{5}$ F2R-Lv-infected Hepa1-6 cells and then subjected to PD-1 mAb treatment or receiving lgG2a isotype control. A A schematic representation of the treatment plan for immune-competent C57BL/6 mice. B Plots of Hepa1-6 tumor volumes, which were recorded once a week. C, D $B A L B / c$ nude mice, injected with GZMA-rAd or RFP-rAd-infected CD ${ }^{+}$T cells, were injected with $2 \times 10^{5}$ F2R-Lv-infected Huh7 cells and then subjected to PD-1 mAb treatment or receiving the IgG2a isotype control. C A schematic representation of the treatment plan for immune-deficient $B A L B / C$ nude mice. D Plots of tumor volumes, which were recorded once a week. E Representative images of the immunohistochemistry staining of GZMA and F2R expressions in the tumor samples from HCC patients. Scale bars: 50 mm. $\mathbf{F}$ The densities of GZMA and F2R in PD-1 mAb responder and non-responder HCC patients. G Tumor diameters recorded by a radiologist based on CT imaging, indicated with a red line. $\mathbf{H}$ The changed tumor diameter $(\mathrm{mm})$ in the HCC patients treated with PD-1 mAb. The tumors were with an increased diameter are indicated in red, while the tumors with a decreased diameter are indicated in green. I Spearman's rank correlation analysis was performed to determine the quantitative correlation between the tumor diameter change and the GZMA and F2R expression levels. The results were expressed as mean $\pm \mathrm{SEM} ;{ }^{*} p<0.05,{ }^{*} p<0.01,{ }^{* * *} p<0.001,{ }^{* * * *} p<0.0001$.

injected into the mice via the tail vein once a week. Tumor diameter was recorded weekly. Later, the animal was sacrificed, a frozen tissue section was constructed, and the TUNEL assay and activated caspase 3 staining were performed.

\section{Immunoblotting}

Tumor tissues or cell pellets were homogenized using cold lysis buffer procured from Solarbio Life Sciences. The homogenates were centrifuged at $8,000 \mathrm{~g}$ for $30 \mathrm{~min}$. After BCA quantification, the supernatant was separated using SDS-PAGE gels, and the separated proteins were transferred to PVDF membranes. The membranes with the proteins were incubated separately with GZMA, F2R, JAK2, p-JAK2, STAT1, Tubulin, and GAPDH antibodies (1:1 000 dilution). After the incubation, the membranes were washed with 1xTBST and then incubated with the HRP-conjugated secondary antibodies (1:2 000 dilution). Afterward, the membrane was washed again with 1xTBST and then incubated with $1 \mathrm{ml}$ of the electrochemiluminescence solution. The protein bands were visualized using the Tanon image system. The regents and antibody clones that were used are listed in Table S7. Original western blots are available in supplemental data.

\section{Nuclear extraction}

The nuclear extraction assay was performed according to the protocol provided by the manufacturer [52]. Briefly, cells were collected in a 15-ml tube and centrifuged at $400 \mathrm{~g}$. The obtained cell pellet was collected and resuspended in $100 \mu \mathrm{L}$ of the pre-extraction buffer. After $10 \mathrm{~min}$, the cytoplasmic extract obtained was transferred to a fresh EP tube and then centrifuged at $8,000 \mathrm{~g}$. The resulting nuclear pellet was mixed with an extraction buffer, and the mixture was incubated on ice for $15 \mathrm{~min}$. Subsequently, the mixture was sonicated in an ultrasonic disintegrator for $3 \times 10 \mathrm{sec}$. Afterward, the sonicated mixture was centrifuged a $12,000 \mathrm{~g}$ for $10 \mathrm{~min}$, and the nuclear extracts obtained were transferred to a fresh EP tube. Finally, immunoblotting was performed as described earlier.

\section{Cell counting kit-8 assays}

The cck8 assay was performed according to the protocol provided by the manufacturer [53]. Briefly, 2,000 cells were plated in a 96-well tissue culture plate for $8 \mathrm{~h}$ and then co-cultured with $8000(1: 4) \mathrm{CD}^{+} \mathrm{T}$ cells. At the end of the assay, the cells were incubated with $100 \mu \mathrm{l}$ of a medium supplemented with $10 \mu \mathrm{l}$ of the CCK-8 solution for $1 \mathrm{~h}$. The $450 \mathrm{~nm}$ absorbance was recorded at $450 \mathrm{~nm}$ using a microplate reader.

\section{T cell-mediated tumor cell killing assay}

The T cell-mediated tumor cell killing assay was performed as described in the report by of Hong L [54]. Briefly, the HepG2 and Huh7 cells were plated in a 96-well tissue culture plate for $8 \mathrm{~h}$ and then co-cultured with $\mathrm{CD}^{+}$ $\mathrm{T}$ cells for $48 \mathrm{~h}$. After removing the T cells and other cell debris, the remaining cells were stained with crystal violet and then quantified at $570 \mathrm{~nm}$ in a spectrometer.

\section{TUNEL assay}

The terminal deoxynucleotidyl transferase dUTP nick-end labeling assay was performed as described in a previous report [55]. Briefly, the tissue slices were fixed using $70 \%$ alcohol and penetrated with $0.5 \%$ Triton X-100. The DNA $3^{\prime}-\mathrm{OH}$ in the apoptotic cells was linked with the FITC-labeled dUTP. The DNA was stained with DAPI, and the FITC-positive cell were visualized under a fluorescence microscope.

\section{Calcein-AM/PI double staining assay}

HepG2 and Huh7 cells were plated in a 24-well tissue culture plate and then co-cultured with $\mathrm{CD}^{+}$T cells for $48 \mathrm{~h}$. Subsequently, the staining of apoptotic cells was performed through incubation with propidium iodide at $37^{\circ} \mathrm{C}$ for $15 \mathrm{~min}$. Next, the living cells were stained using Calcein AM. The $\mathrm{PI}$ and Calcein AM-positive cells were visualized using fluorescence microscopy.

\section{Fluorescence confocal assay}

The cultured cells or tissue slices were fixed using $75 \%$ alcohol and penetrated with $0.5 \%$ Triton $\mathrm{X}-100$. This was followed by incubation with GZMA, F2R, STAT1, and activated-casp3. Afterward, the samples were washed with $1 \times$ PBS and then incubated with FITC or TRITC-labeled secondary antibody (1:400 dilution). Subsequently, after another wash with $1 x$ PBS, the samples were stained with DAPI and covered with a cover glass. Finally, the protein was visualized and photographed under a fluorescence microscope.

\section{Total RNA extraction}

Tissues were homogenized using the TRlzol reagent. The lysed tissue homogenate was centrifuged at $3,000 \mathrm{~g}$, and the pellet was discarded. The obtained supernatant was incubated with chloroform and followed by thorough mixing and then centrifuged at $12000 \mathrm{~g}$. The supernatant was collected, and isopropanol was added to it for RNA precipitation. The precipitate containing the RNA was again centrifuged at $12,000 \mathrm{~g} .70 \%$ ethanol was used to wash the RNA and collected at $8,000 \mathrm{~g}$. The precipitated RNA was finally dissolved in RNase-free water.

\section{CDNA synthesis}

The CDNA was synthesized using the SuperScript ${ }^{\circledR}$ III First-Strand Synthesis kit for RT-qPCR. Total RNA $(\leq 2.5 \mu \mathrm{g})$, dNTP mix $(1 \mathrm{mM})$, and random hexamer primers $(5 \mathrm{ng} / \mu \mathrm{l})$ were mixed, followed by the addition of water to attain a final volume of $5 \mu \mathrm{l}$. RT buffer, RNaseOUT (2 U), DDT (10 mM), SuperScript ${ }^{\circ} \mathrm{II}(10 \mathrm{U})$, and $\mathrm{MgCl}_{2}(5 \mathrm{mM})$ were mixed, forming a total volume of $10 \mu \mathrm{l}$. Subsequently, a standard CDNA synthesis program was run, and the synthesized cDNA was stored at $-20^{\circ} \mathrm{C}$.

\section{qRT-PCR analysis}

The primers, Sybr Green, and the synthesized CDNA were mixed and briefly centrifuged. Next $3.8 \mu \mathrm{l}$ of $\mathrm{ddH}_{2} \mathrm{O}, 1 \mu \mathrm{l}$ of cDNA, $5 \mu \mathrm{l}$ of Sybr Green, and $0.2 \mu \mathrm{l}$ of primer $(10 \mu \mathrm{M})$ were added to a 384 -well PCR plate. The PCR program conditions used were: denaturation at $95^{\circ} \mathrm{C}$ for $15 \mathrm{~s}$, annealing at $56{ }^{\circ} \mathrm{C}$ for $30 \mathrm{~s}$, elongation at $72{ }^{\circ} \mathrm{C}$ for $50 \mathrm{~s}$; the number of cycles run was 40 . The housekeeping gene GAPDH was used as the internal standard. The primer pairs used are provided in Table S5.

\section{Data and statistical analyses}

The high-quality cells were separated by applying the following criteria: nFeature_RNA $>500$ \& nCount_RNA $>1000$ \& nCount_RNA $<20000$ \& percent.mt $<15$. The FindClusters function was run to identify the cell clusters at a resolution of 0.5. The Human Cell Atlas Data (http://biocc. hrbmu.edu.cn/CellMarker/) was employed for the annotation of the identified cell clusters based on marker genes. RNA-Seq of tumor tissues and adjacent tissues data were downloaded from TCGA. The GSEA pathway analysis was performed based on the marker gene.

Statistical analyses were performed using IBM SPSS statistic 22 (IBM, New York, USA). The differences among the variables were determined using the 

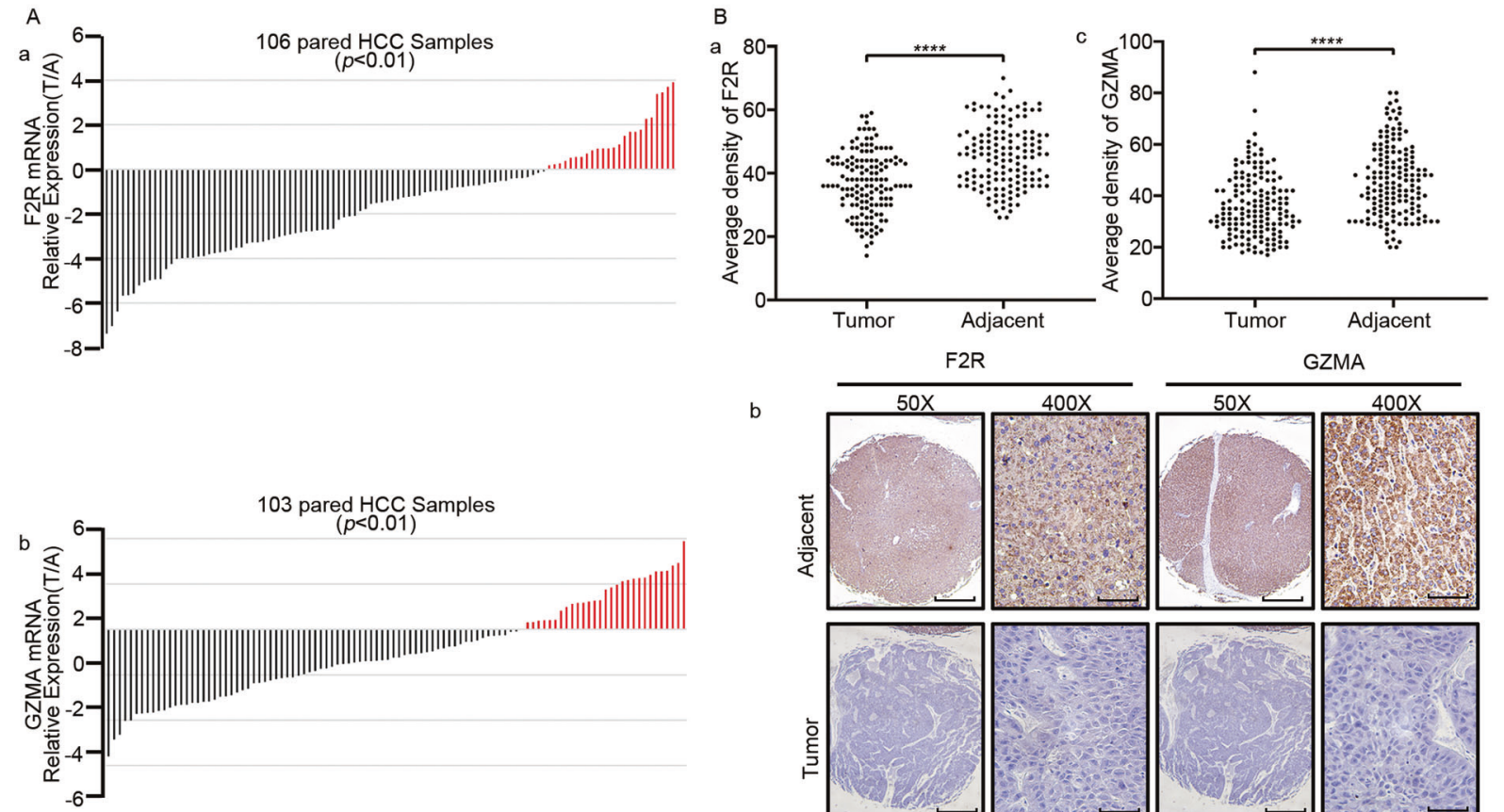

b
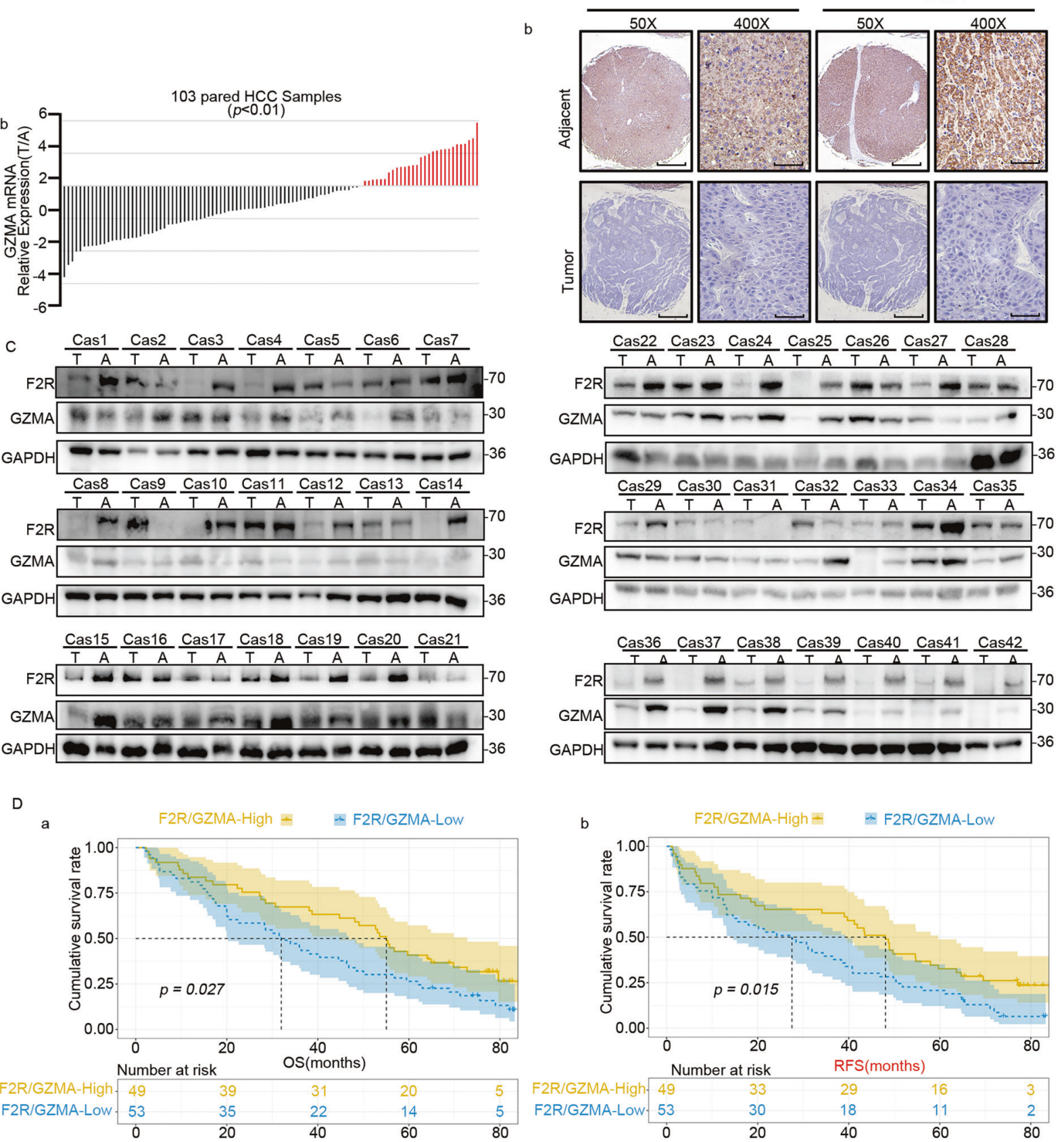

Fig. 8 Downregulation of the GZMA and F2R expressions predicted aggressive clinicopathological characteristics and poor prognosis in HCC patients. A The mRNA levels of GZMA and F2R in 106 pairs of tumor tissues and adjacent tissues from HCC patients, analyzed using qRTPCR. The relative GZMA and F2R expression were normalized using GAPDH $\left(^{-\Delta \Delta C T}\right.$ ). B IHC staining was performed for GZMA and F2R in 158 pairs of tumor tissues and adjacent tissues from HCC patients. Scale bars: $2 \mathrm{~mm}$ for 50x and $50 \mu \mathrm{m}$ for $400 x$. The average intensity of the gray color was used to indicate the relative levels of GZMA and F2R. ${ }^{*} p<0.05,{ }^{*} p<0.01,{ }^{* * *} p<0.001,{ }^{* * * *} p<0.0001$. C The protein levels of GZMA and F2R in 42 pairs of tumor tissues and adjacent tissues from HCC patients were semi-quantified using the western blot assay. D The overall and tumor-free survival assays were performed for 106 low GZMA/F2R expression and high GZMA/F2R expression HCC patients. The patients at risk are listed below. 
two-tailed student's t-test. The log-rank test was used for determining the progression-free, disease-free, overall, and disease interval survival. A stepwise Cox multivariate proportional hazard regression model was used for the multivariate analysis. Statistical significance was demonstrated by the $P$ values of 0.05 . The data were presented as mean \pm SD.

\section{DATA AVAILABILITY}

The datasets used and/or analyzed in the present study are available with the corresponding author and would be provided for study upon reasonable request.

\section{REFERENCES}

1. Saleh R, Toor SM, Nair VS, Elkord E. Role of epigenetic modifications in inhibitory immune checkpoints in cancer development and progression. Front Immunol. 2020;11:1469.

2. Sui H, Ma N, Wang Y, Hui L, Liu X, Su Y, et al. Anti-PD-1/PD-L1 therapy for nonsmall-cell lung cancer: toward personalized medicine and combination strategies. J Immunol Res. 2018;2018:1-17.

3. Hugo W, Zaretsky JM, Sun L, Song CY, Moreno BH, Hu-Lieskovan S, et al. Genomic and transcriptomic features of response to anti-PD-1 therapy in metastatic melanoma. Cell. 2017;168:542.

4. Voorwerk L, Slagter M, Horlings HM, Sikorska K, van de Vijver KK, de Maaker M, et al. Immune induction strategies in metastatic triple-negative breast cancer to enhance the sensitivity to PD-1 blockade: the TONIC trial. Nat Med. 2019;25:920-8.

5. Voutsadakis IA. International PD: PD-1 inhibitors monotherapy in hepatocellular carcinoma: meta-analysis and systematic review. Hepatobiliary Pancreat Dis Int. 2019;18:505-10

6. Skoulidis F, Goldberg ME, Greenawalt DE, Hellmann MD, Awad MM, Gainor JF, et al. STK11/LKB1 mutations and PD-1 inhibitor resistance in KRAS-mutant lung adenocarcinoma. Cancer Disco. 2018;8:822-35.

7. Galarreta D, Bresnahan E, Molina-Sánchez P, Lindblad KE, Barbara Maier B, Sia D, et al. Barbara: beta-catenin activation promotes immune escape and resistance to Anti-PD-1 therapy in hepatocellular carcinoma. Cancer Disco. 2019;9:1124-41.

8. Hao JJ, Lin D, Dinh HQ, Mayakonda A, Jiang YY, Chang C, et al. Spatial intratumoral heterogeneity and temporal clonal evolution in esophageal squamous cell carcinoma. Nat Genet. 2016;48:1500-7.

9. Du K, Wei SY, Wei Z, Frederick DT, Miao BC, Moll T, et al. Pathway signatures derived from on-treatment tumor specimens predict response to anti-PD1 blockade in metastatic melanoma. Nat Commun. 2021;12:6023.

10. Kenichi S, Jihye K, Isao M, Leslie R, Masaki S, Shigeki S, et al. Innate genetic evolution of lung cancers and spatial heterogeneity: analysis of treatment-nave lesions. J Thorac Oncol. 2018;13:1496-507.

11. Chamseddine IM, Rejniak KA. Hybrid modeling frameworks of tumor development and treatment. Wiley Interdiscip Rev Syst Biol Med. 2020;12:e1461.

12. Vitale I, Manic G, Coussens LM, Kroemer G, Galluzzi LJ. Macrophages and metabolism in the tumor microenvironment. Cell Metab. 2019;30:36-50.

13. Liu T, Zhou L, Li D, Andl T, Zhang Y. Cancer-associated fibroblasts build and secure the tumor microenvironment. Front Cell Dev Biol. 2019;7:60.

14. Eigentler TK, Hassel JC, Berking C, Aberle J, Bachmann O, Grünwald V, et al. Diagnosis, monitoring and management of immune-related adverse drug reactions of anti-PD-1 antibody therapy. Cancer Treat Rev. 2016;45:7-18.

15. Schneider BJ, Naidoo J, Santomasso BD, Lacchetti C, Adkins S, Anadkat M, et al. Management of immune-related adverse events in patients treated with immune checkpoint inhibitor therapy: ASCO guideline update. J Clin Oncol. 2021;39:4073-126.

16. Gutierrez-Rodriguez M, Herranz R. From multiple PAR1 receptor/protein interactions to their multiple therapeutic implications. Curr Top Med Chem. 2015;15:2080-114.

17. Pompili E, Franchis VD, Giampietri C, Leone S, Santis ED, Fornai F, et al. Protease activated receptor 1 and its ligands as main regulators of the regeneration of peripheral nerves. Biomolecules. 2021;11:1668.

18. Han X, Nieman MT, Kerlin BA. Protease-activated receptors: an illustrated review. Res Pr Thromb Haemost. 2020;5:17-26.

19. Chang YH, Wu JC, Yu HM, Hsu HT, Wu YT, Yu ALT, et al. Design and synthesis of glyco-peptides as anti-cancer agents targeting thrombin-protease activated receptor-1 interaction. Chem Commun (Camb). 2020;56:5827-30.

20. Ray T, Pal A. PAR-1 mediated apoptosis of breast cancer cells by V. cholerae hemagglutinin protease. Apoptosis. 2016;21:609-20.

21. Wojtukiewicz MZ, Hempel D, Sierko E, Tucker SC, Honn KV. Endothelial Protein C Receptor (EPCR), Protease Activated Receptor-1 (PAR-1) and Their Interplay in Cancer Growth and Metastatic Dissemination. Cancers (Basel). 2019;11:51.

22. Zeglinski MR, Granville DJ. Granzymes in cardiovascular injury and disease. Cell Signal. 2020;76:109804.
23. Trapani JA. Granzymes, cytotoxic granules and cell death: the early work of Dr. Jurg Tschopp. Cell Death Differ. 2012;19:21-7.

24. Déry O, Corvera CU, Steinhoff M, Bunnett NW. Proteinase-activated receptors: novel mechanisms of signaling by serine proteases. Am J Physiol. 1998;274:1429-52.

25. Cabral L, Tiribelli C, Sukowati C. Sorafenib resistance in hepatocellular carcinoma: the relevance of genetic heterogeneity. Cancers (Basel). 2020;12:1576.

26. Chiu KC, Yuen WH, Cheu WS, Wei LL, Ting V, Fehlings $M$, et al. Hepatocellular carcinoma cells up-regulate PVRL1, stabilizing PVR and inhibiting the cytotoxic T-cell response via TIGIT to mediate tumor resistance to PD1 inhibitors in mice. Gastroenterology. 2020;159:609-23.

27. Zhang S, Zeng C, Wang D, Gao X, Guo S, Zhang Y, et al. Efficient induction of cytotoxic T lymphocytes in hepatocellular carcinoma using the HLA-A2-restricted survivin peptide in vitro. Exp Cell Res. 2020;386:111741.

28. Meng FZ, Zhen SM, Song B. HBV-specific CD4+ cytotoxic T cells in hepatocellular carcinoma are less cytolytic toward tumor cells and suppress CD8 $+\mathrm{T}$ cellmediated antitumor immunity. APMIS. 2017;125:743-51.

29. Wesch D, Kabelitz D, Oberg HH. Tumor resistance mechanisms and their consequences on $\gamma \delta$ T cell activation. Immunol Rev. 2020;298:84-98.

30. Mel SD, Hue SS, Jeyasekharan AD, Chng WJ, Ng SB. Molecular pathogenic pathways in extranodal NK/T cell lymphoma. J Hematol Oncol. 2019;12:33.

31. Dermani FK, Samadi P, Rahmani G, Kohlan AK, Najafi R. PD-1/PD-L1 immune checkpoint: Potential target for cancer therapy. J Cell Physiol. 2019;234:1313-25.

32. Ai L, Chen J, Yan H, He QJ, Luo PH, Xu ZF, et al. Research Status and Outlook of PD-1/PD-L1 Inhibitors for Cancer Therapy. Drug Des Devel Ther. 2020;14:3625-49.

33. Griggio V, Perutelli $F$, Salvetti $C$, Boccellato $E$, Boccadoro $M$, Vitale $C$, et al. Immune dysfunctions and immune-based therapeutic interventions in chronic lymphocytic leukemia. Front Immunol. 2020;11:594556.

34. Melaiu O, Lucarini V, Giovannoni R, Fruci D, Gemignani F. News on immune checkpoint inhibitors as immunotherapy strategies in adult and pediatric solid tumors. Semin Cancer Biol. 2020;S1044-579X:30156-5.

35. Qin S, Xu L, Yi M, Yu S, Wu K, Luo S. Novel immune checkpoint targets: Moving beyond PD-1 and CTLA-4. Mol Cancer. 2019;18:155.

36. Mehdizadeh S, Bayatipoor H, Pashangzadeh S, Jafarpour R, Shojaei Z, Motallebnezhad M. Immune checkpoints and cancer development: therapeutic implications and future directions. Pathol Res Pr. 2021;223:153485.

37. Pérez-Ruiz E, Melero I, Kopecka J, Sarmento-Ribeiro AB, Marilina García-Aranda M, Rivas JDL. Cancer immunotherapy resistance based on immune checkpoints inhibitors: Targets, biomarkers, and remedies. Drug Resist Updat. 2020;53:100718.

38. Lei $Q$, Wang D, Sun K, Wang L, Zhang Y. Resistance mechanisms of anti-PD1/PDL1 therapy in solid tumors. Front Cell Dev Biol. 2020;8:672.

39. Sun C, Mezzadra R, Schumacher TN. Regulation and Function of the PD-L1 Checkpoint. Immunity. 2018;48:434-52.

40. Makuku R, Khalili N, Razi S, Keshavarz-Fathi M, Rezaei N. Current and future perspectives of PD-1/PDL-1 blockade in cancer immunotherapy. J Immunol Res. 2021:4:1-15.

41. Wang $Z Y$, Wu XY. Study and analysis of antitumor resistance mechanism of PD1/ PD-L1 immune checkpoint blocker. Cancer Med. 2020;9:8086-121.

42. Schweickert PG, Yang Y, White EE, Cresswell GM, Elzey BD, Ratliff TL. ThrombinPAR1 signaling in pancreatic cancer promotes an immunosuppressive microenvironment. J Thromb Haemost. 2021:19:161-72.

43. Zigler M, Kamiya T, Brantley EC, Villares GJ, Bar-Eli M. PAR-1 and thrombin: the ties that bind the microenvironment to melanoma metastasis. Cancer Res. $2011 ; 71: 6561-6$.

44. Heider I, Schulze B, Oswald E, Henklein P, Scheele J, Kaufmann R. PAR1-type thrombin receptor stimulates migration and matrix adhesion of human colon carcinoma cells by a PKCepsilon-dependent mechanism. Oncol Res. 2004; 14:475-82.

45. Willis Fox O, Preston RJ. Molecular basis of protease-activated receptor 1 signaling diversity. J Thromb Haemost. 2020;18:6-16.

46. Posma JJ, Grover SP, Hisada Y, Owens AP 3rd, Antoniak S, Spronk HM, et al. Roles of coagulation proteases and PARs (protease-activated receptors) in mouse models of inflammatory diseases. Arterioscler Thromb Vasc Biol. 2019;39:13-24.

47. Suidan HS, Clemetson KJ, Brown-Luedi M, Niclou SP, Clemetson JM, Tschopp J. The serine protease granzyme $A$ does not induce platelet aggregation but inhibits responses triggered by thrombin. Biochem J. 1996;315:939-45.

48. Arias M, Martínez-Lostao L, Santiago L, Ferrandez A, Granville DJ, Pardo J. The untold story of granzymes in oncoimmunology: novel opportunities with old acquaintances. Trends Cancer. 2017;3:407-22.

49. Wong PS, Sutejo R, Chen H, Ng SH, Sugrue RJ, Tan BH. A system basedapproach to examine cytokine response in poxvirus-infected macrophages. Viruses. 2018;10:692.

50. Yang TW, Ouyang YB, Gao YX, Liu DJ, Zang YJ, Chen DX. Enriched highthroughput reverse transcriptionquantitative PCR template preparation without preamplification. Mol Med Rep. 2020;22:3541-8. 
14

51. Wang $X C, H e Q F$, Shen $H Y$, Xia AL, Tian WF, Yu WW, et al. TOX promotes the exhaustion of antitumor CD8+ T cells by preventing PD1 degradation in hepatocellular carcinoma. J Hepatol. 2019;71:731-41.

52. Li LC, Zhao S, Liu ZF, Zhang NZ, Pang S, Liu JK, et al. Sunitinib treatment promotes metastasis of drug-resistant renal cell carcinoma via TFE3 signaling pathway. Cell Death Dis. 2021;12:220.

53. Yu WH, Chen K, Ye GW, Wang S, Wang P, Li JT, et al. SNP-adjacent super enhancer network mediates enhanced osteogenic differentiation of MSCs in ankylosing spondylitis. Hum Mol Genet. 2021;30:277-93.

54. Liu $\mathrm{H}$, Kuang XW, Zhang YC, Ye YQ, Li JL, Liang L, et al. ADORA1 inhibition promotes tumor immune evasion by regulating the ATF3-PD-L1 axis. Cancer Cell. 2020;37:324-39.

55. Yang TW, Gao YX, Liu DJ, Wang Y, Wu J, Liu XN, et al. ASPP2 enhances chemotherapeutic sensitivity through the down-regulation of XIAP expression in a p53 independent manner in hepatocellular carcinoma. Biochem Biophys Res Commun. 2019;508:769-74.

\section{ACKNOWLEDGEMENTS}

We are grateful to the organizers and participants of the TCGA and GTEx project, and Lu Y, Beijing Institute of Radiation Medicine, for the single-cell RNA-Seq data available in GEO, which were used in the present study.

\section{AUTHOR CONTRIBUTIONS}

TY, YG, QX, XL, YG, BZ, YJ, CZ, YS, PY, and RJ contributed to data research. TY, QX, YS, $\mathrm{DL}, \mathrm{XL}$, and $\mathrm{DC}$ contributed designing the study. TY, YG, YG, QX, YS, XL, YO, WW, QX, and $\mathrm{RJ}$ assisted in report preparation. TY and DC supervised the study. All authors have thoroughly read and approved the final manuscript.

\section{FUNDING}

The present research work was supported by the National Natural Science Foundation of China (82073676, 81470098, 81900575), the Beijing Municipal Commission of Science and Technology (Z191100006619064), the Beijing Municipal Natural Science Foundation, and the Beijing Municipal Education Commission (KZ202010025037).
COMPETING INTERESTS

The authors declare no competing interests.

\section{CONSENT TO PARTICIPATE}

Samples were obtained with informed consent.

\section{ADDITIONAL INFORMATION}

Supplementary information The online version contains supplementary material available at https://doi.org/10.1038/s41419-022-04654-7.

Correspondence and requests for materials should be addressed to Tongwang Yang.

Reprints and permission information is available at http://www.nature.com/ reprints

Publisher's note Springer Nature remains neutral with regard to jurisdictional claims in published maps and institutional affiliations.

cc) (i)

Open Access This article is licensed under a Creative Commons Attribution 4.0 International License, which permits use, sharing, adaptation, distribution and reproduction in any medium or format, as long as you give appropriate credit to the original author(s) and the source, provide a link to the Creative Commons license, and indicate if changes were made. The images or other third party material in this article are included in the article's Creative Commons license, unless indicated otherwise in a credit line to the material. If material is not included in the article's Creative Commons license and your intended use is not permitted by statutory regulation or exceeds the permitted use, you will need to obtain permission directly from the copyright holder. To view a copy of this license, visit http://creativecommons. org/licenses/by/4.0/.

(c) The Author(s) 2022 